\title{
Parental decision-making following a prenatal diagnosis that is lethal, life- limiting, or has long term implications for the future child and family: a meta- synthesis of qualitative literature
}

\author{
Claire Blakeley ${ }^{1,2}$, Debbie M. Smith ${ }^{1,3}$, Edward D. Johnstone $e^{1,4}$ and Anja Wittkowski, ${ }^{1,2^{*}}$ (D)
}

\begin{abstract}
Background: Information on the factors influencing parents' decision-making process following a lethal, life-limiting or severely debilitating prenatal diagnosis remains deficient. A comprehensive systematic review and meta-synthesis was conducted to explore the influencing factors for parents considering termination or continuation of pregnancy following identification of lethal, life-limiting or severely debilitating fetal abnormalities.

Methods: Electronic searches of 13 databases were conducted. These searches were supplemented by hand-searching Google Scholar and bibliographies and citation tracing. Thomas and Harden's (2008) thematic synthesis method was used to synthesise data from identified studies.

Results: Twenty-four papers were identified and reviewed, but two papers were removed following quality assessment. Three main themes were identified through systematic synthesis. Theme 1, entitled 'all life is precious', described parents' perception of the importance of the fetus' life, a fatalistic view of their situation alongside moral implications as well as the implications decisions would have on their own life, in consideration of previous life experiences. Theme 2 ('hope for a positive outcome') contained two sub-themes which considered the parent's own imagined future and the influence of other people's experiences. Finally, Theme 3 ('a life worth living') presented three sub-themes which may influence their parental decision-making: These described parental consideration of the quality of life for their unborn child, the possibility of waiting to try for another pregnancy, and their own responsibilities and commitments.

Conclusion: The first review to fully explore parental decision-making process following lethal, life-limiting, or severely debilitating prenatal diagnosis provided novel findings and insight into which factors influenced parents' decision-making process. This comprehensive and systematic review provides greater understanding of the factors influential on decisionmaking, such as hope, morality and potential implications on their own and other's quality of life, will enable professionals to facilitate supported decision-making, including greater knowledge of the variables likely to influence parental choices.
\end{abstract}

Keywords: Termination, Continuation, Pregnancy, Life-limiting, Abortion, Birth, Disorder

\footnotetext{
* Correspondence: Anja.wittkowski@manchester.ac.uk

${ }^{1}$ The University of Manchester, Faculty of Biology, Medicine and Health,

School of Health Sciences, Division of Psychology and Mental Health, 2nd

Floor, Zochonis Building, Brunswick Street, Manchester M13 9PL, UK

${ }^{2}$ Greater Manchester Mental Health Foundation Trust, Trust Headquarters,

Bury New Road, Prestwich, Manchester M25 3BL, UK

Full list of author information is available at the end of the article
}

(c) The Author(s). 2019 Open Access This article is distributed under the terms of the Creative Commons Attribution 4.0 International License (http://creativecommons.org/licenses/by/4.0/), which permits unrestricted use, distribution, and reproduction in any medium, provided you give appropriate credit to the original author(s) and the source, provide a link to the Creative Commons license, and indicate if changes were made. The Creative Commons Public Domain Dedication waiver (http://creativecommons.org/publicdomain/zero/1.0/) applies to the data made available in this article, unless otherwise stated. 


\section{Plain English summary}

Little is known about how parents make choices after finding out their unborn child has a disorder or illness which might seriously affect their chance of surviving or increase their likelihood of having a severe disability after birth. For this reason, we undertook a review of studies that help us understand how parents make decisions.

To perform our analysis we searched 13 different databases, Google Scholar and reference lists. We chose to include only qualitative studies in our review. We reviewed papers resulting from these studies using a method described by Thomas and Harden (2008). The search identified 24 papers which discussed how parents make decisions after being told their unborn baby had a serious illness but two papers were removed because they were poor. The final 22 papers revealed that parents had many thoughts about whether to carry on with or end their pregnancy. These thoughts included how special the pregnancy was to them. They were also hopeful that their pregnancy would end well. Finally, they thought about whether their baby would suffer if he or she survived.

The findings of this review will be helpful for health professionals who look after women and their partners when problems are identified during pregnancy. We offer new insights into why and how parents reach decisions they do about what course of action they take.

\section{Introduction}

The identification of lethal, life-limiting, or severely debilitating fetal abnormalities prenatally has increased over the past few decades, with prenatal screening standard practice in many countries [1]. Approximately 2-3\% of all parents attending prenatal clinics for screening receive results suggestive of fetal abnormality [2, 3], leaving them with emotional decisions to make in relation to the continuation or termination of pregnancies $[4,5]$. Between 81 and $90 \%$ of parents terminate pregnancies identified with lethal, life-limiting, or severely debilitating disorders [6-8], whilst others choose to continue with their pregnancy. However, no concise review detailing all factors influencing parental decision-making when parents are faced with the option to continue or terminate a pregnancy affected by lethal, life-limiting, or severely debilitating prenatal diagnosis has been provided; hence there is a need for further clarification to ensure adequate support is provided during this period.

Parents deciding to continue or terminate a pregnancy following diagnosis of fetal abnormality experience grief, shock [9-11], disbelief, isolation, anger and adaptation or adjustment $[9,10,12]$. Studies have identified several influential factors on parental decision-making following prenatal diagnosis. Expectant parents' observation of ultrasound examinations influenced their attachment to the fetus [13, 14] and a diagnosis of fetal disorder was not found to disrupt this attachment [15]. Advanced maternal age is a contributing factor in attachment and personification of a fetus, especially for women who perceive the pregnancy to be their final chance to give birth [16], with this attachment increasing through the development of the pregnancy $[17,18]$. Religion has also been suggested as a leading factor in decision-making for parents faced with the option to terminate a fetus [18-20]. For example, faith has been found to encourage hopeful and fatalistic beliefs [21], with fear of disobeying religious scripture playing an important role in decision-making [22].

Whilst studies provide examples of factors influencing parental decision-making, they do not infer how maternal healthcare teams working with parents should best support them in making decisions beyond non-judgemental support [23] and counselling for likely outcomes [24], leaving important recommendations to better prepare maternal healthcare teams for this supportive role. The studies discussed were largely quantitative in methodology [16-20], with qualitative studies focussing on hypothetical scenarios and cultural acceptance of termination $[18,22]$ or the experience of mothers in countries where termination is illegal [21]. An exploration of parents' lived experiences following prenatal diagnosis of lethal, life-limiting, or severely debilitating diagnosis provides insight into the thoughts of parents in this unique situation, informing the evidence base of influential factors in parental decisionmaking, and how maternal healthcare teams can facilitate and support parents during this time.

Within their syntheses of parental experiences of prenatal diagnosis, three reviews provided an insight into parental experiences at the time of making a decision [25-27]. In their review of 17 studies, Sandelowski and Barrosso [25] investigated the experience of diagnosis of any prenatal condition following screening for expectant parents in the USA. The authors highlighted some influential factors in decision-making: Parents reported that they combined new information with prior knowledge or beliefs about disability, parenthood and the moral or religious acceptability of abortion when making their decision to terminate or continue an affected pregnancy. Parents considered the certainty of the diagnosis, their ability to parent a child with the disorder and how this would impact others, their prior fertility, support from family and friends, and the pressured timeframe within which to make their decision. Parents also discussed their anticipated hope that their child would be born without the disorder and their perceived guilt around not allowing their pregnancy to continue. This 2005 meta-synthesis was limited in its sample of 17 studies, due to geographical and language biases. The synthesis also included 10 studies, which were unpublished at the time of the review, with risk of reduced quality. 
Lafarge et al.'s meta-ethnography [26] reviewed 14 English language studies which addressed women's experiences of choosing to terminate a pregnancy, following prenatal diagnosis. They identified that women expressed feelings of guilt and hopelessness when deciding to end their pregnancy. However, they also considered the baby's potential quality of life as well as the impact on them and their wider family. The review focussed on the women's experience of the termination process, addressing women's decision-making in two sub-themes, whilst the remaining ten sub-themes focussed on the experience of women. Studies were not excluded from this review if they did not consider parental decision-making.

Lou et al's [27] qualitative systematic review presented findings on parents' experiences of prenatal diagnosis, discussing their relationship with clinicians, other's acknowledgment of their pregnancy and stigmatization experienced. This review of 28 studies of parents within European or English speaking countries highlighted the multiple losses experienced by parents during this difficult time, but it did not address the decision-making process parents are faced with following prenatal diagnosis.

These reviews did not provide a breadth of understanding in relation to factors influencing decision-making in the period following prenatal diagnosis of a lethal, lifelimiting, or severely debilitating condition. In order to guide clinical practice in the support of parents following prenatal diagnosis of lethal, life-limiting, or severely debilitating disorders, more information is required about the decision-making process. Studies have examined decisionmaking as a psychological construct, with normative models, such as Classical Decision-Making Theory [28], aiming to rationalise a complex and individual process [29]. Whilst this model provides insight into how best to approach a decision, it does not necessarily describe how people choose in real-life situations. Descriptive models, such as Prospect Theory [29], provide insight into how individuals make real-life choices. Descriptive models suggest that schemata, encompassing prior life experiences, are drawn on to make decisions [30], considering potential outcomes of each option available to them, and the likelihood of each option occurring [30]. Accoding to EmotionImbued Choice Theory [31], individuals imagine their perceived emotional response to each available outcome as a way of mediating decisions. Individually salient impacting factors, such as culture [32], current emotional state [33], delivery of information [34] and time constraints [35], must be considered when supporting parents making decisions.

Thus, the aim of this review was to explore parental decision-making processes following diagnosis of a lethal, life-limiting, or severely debilitating prenatal diagnosis systematically and highlight areas of recommendation for current maternal healthcare practice.

\section{Methodology}

This systematic review and meta-synthesis of qualitative studies followed PRISMA guidelines [36]. The following databases were searched: Allied \& Complementary Medicine (AMED), EBM Reviews, Econlit, Embase, Global Health Archive, Health and Psychosocial Instruments, Health Management Information Consortium (HMIC), International Pharmaceutical Abstracts, Maternity and Infant Care Database, MEDLINE, The Philosopher's Index, PsychINFO, and Social Policy and Practice. The search was inclusive of all years, retrieving papers from the inception of each database up until 27 October 2017. Electronic database searches were supplemented by individual searches of recent editions of relevant journals as well as Google scholar. The review aimed at being inclusive of all papers meeting the inclusion and exclusion criteria outlined in Table 1.

The systematic search process is illustrated in Fig. 1. No new articles were highlighted for inclusion when the search was updated on $22^{\mathrm{n}}$ June 2018.

A check of $10 \%$ of titles, abstracts and full papers reviewed in the search process was conducted by an independent researcher.

\section{Critical appraisal}

All included papers were assessed for quality using the widely used Critical Appraisal Skills Programme (CASP) checklist [37]. As this checklist does not propose a scoring system for which to interpret the quality of each study, the Health Evidence Checklist scoring was also used [38].

\section{Meta-synthesis}

The thematic synthesis approach described by Thomas and Harden [39] was utilised to identify themes presented within the included studies. Building on the concept of thematic analysis, this approach allows for results from different methods of qualitative analysis to be synthesised [40]. The synthesis was implemented in three stages of analysis described by Thomas and Harden [39]. Stage 1 of the process involved both the first author $(\mathrm{CB})$ and second author (DMS) reading each of the included studies a number of times, before independently implementing line-by-line coding within the texts of the results or findings sections of each paper in order to develop codes describing the meaning or content. This process was repeated for each included study, enabling the translation of concepts between the studies as well as identification of new themes. This approach enabled a higher level of analysis to be conducted at stage 2, when both researchers grouped together codes to form descriptive themes apparent across and between papers. The third and final analytical stage of interpreting consistent and inconsistent themes within papers enabled the presentation of analytical themes and sub-themes. 
Table 1 Inclusion and exclusion criteria

Inclusion criteria

Exclusion criteria

Parents who experienced prenatal diagnosis of lethal, life-limiting,

or severely debilitating disorder following prenatal screening

Studies examining or considering in any manner parents' decision-making and the factors influencing their decision following the diagnosis of a

lethal, life-limiting, or severely debilitating disorder, prior to termination or birth

Decisions relating to:

Pre-implantation genetic screening

Acceptance of screening

Type of termination

Choices after termination of pregnancy

Choices following birth of child

Studies which used qualitative methods for data collection and analysis

Studies which captured only quantitative data or used quantitative analysis

Studies written in any language

Primary research

Book reviews, opinion pieces, conference posters or abstracts, literature reviews.
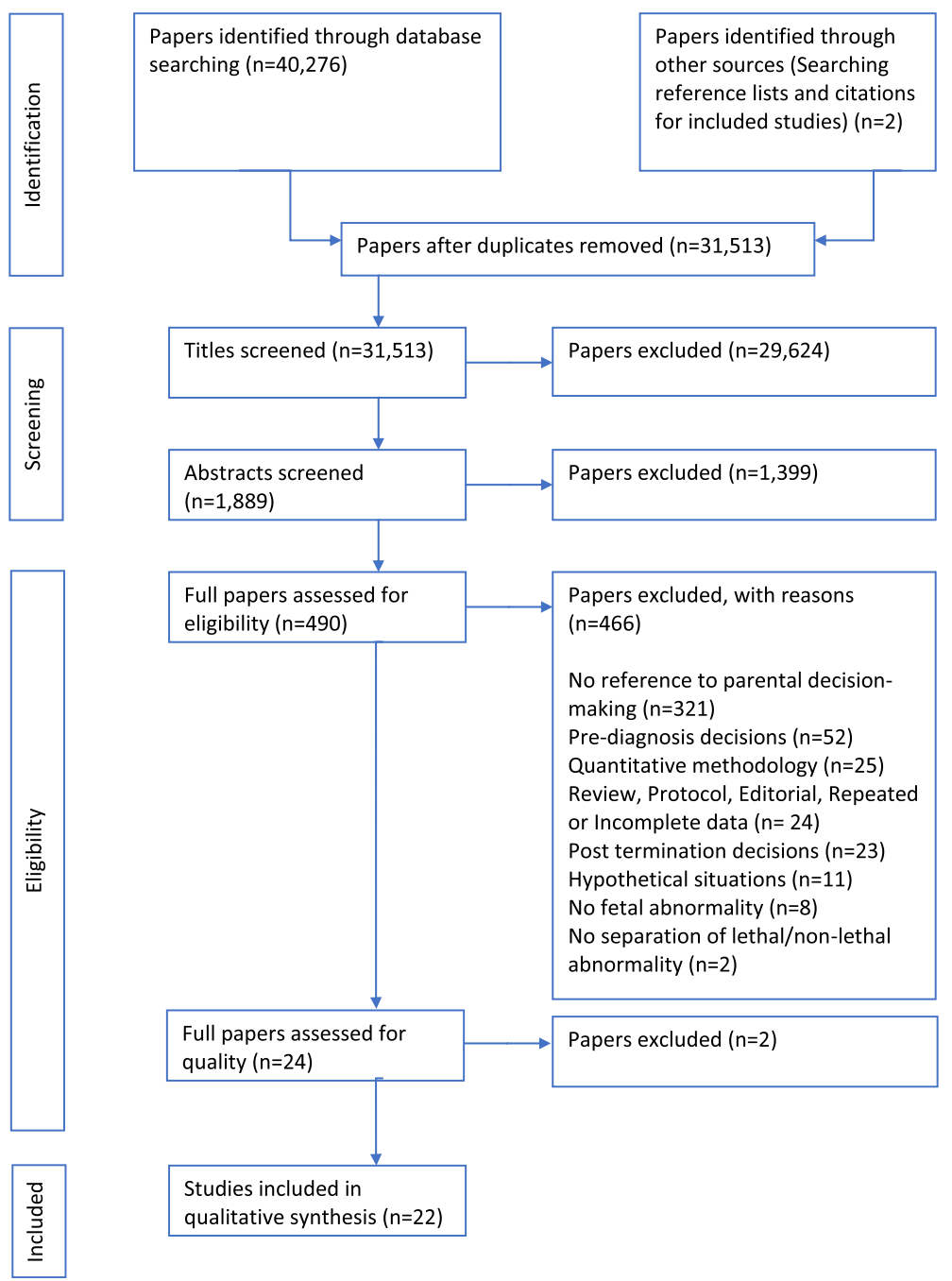

Full papers assessed for quality $(n=24)$

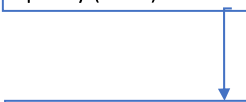

Papers excluded $(n=2)$

Studies included in

qualitative synthesis $(n=22)$

Fig. 1 PRISMA flow diagram, representing the study selection process 
This final stage relied on researcher inference and judgement regarding the meaning behind each code. To ensure reliability, both researchers coded and grouped themes independently, before discussing their views with each other and forming the final themes which were then discussed with and agreed by all authors.

\section{Results}

\section{Quality rating}

The majority $(n=19)$ of the initial 24 included studies were judged to be of low bias and high methodological quality. Three studies [41-43] were rated to be of acceptable quality, which indicated these studies had a moderate risk of bias. Two studies $[44,45]$ were excluded from this review due to risks of low quality and high methodological bias, leaving a final 22 papers for the synthesis. Studies are presented in Table 2, grouped by the decision-making of parents who continued pregnancies, terminated pregnancies or explored both choices.

\section{Themes}

Three themes reflected aspects of influencing parental decision-making following a diagnosis of lethal, lifelimiting, or severely debilitating condition: 1) 'All life is precious', 2) 'Hope for a positive outcome' and 3) 'A life worth living'. These themes encompassed the main areas of decision-making for parents included within this review, with sub-themes present within each theme. The themes, their ten sub-themes, their relation to one another and the process of decision-making are depicted in Fig. 2. Quotes are used within the text to represent the themes.

\section{Theme 1: all life is precious}

A common theme throughout the studies was that parents viewed their fetus as precious: they considered their "baby's worth" [47] and valued the potential life they might have (sub-theme 1). They also considered their own moral and religious beliefs and were guided into doing 'The right thing' (sub-theme 2) based on these judgements. Parents drew on their past-experience of pregnancy or terminations when considering whether they were able to continue or end a pregnancy, and described their views of life with disability and prior experience of this (sub-theme 3). Many studies reported that parents considered their own life pressures and circumstances which could have influenced their decision-making. They considered their beliefs about becoming a parent, with the anticipation or longing for the parent role (sub-theme 4). Finally, sub-theme 5 presented the fatalistic attitude parents described when considering the choices available to them, and whether they were able or fated to continue with a pregnancy that had "happened for a reason" [47].
Sub-theme 1: precious baby Parents described how their connection with their unborn baby influenced their decision-making. They explained how processes such as seeing the baby's face and fetal movement through ultrasounds had personified the baby, with the length of gestation adding to the sense of attachment. Another woman described her previous difficulties in conceiving, and as she had "tried so hard" [47] she was not able to consider ending the pregnancy. Often parents recognised that their attachment to their unborn baby influenced their decisions, and as a result they attempted to reduce the bond with the fetus, allowing them to then make a decision without influence from connection: "I was already 5 months pregnant with JayLynn so she was already a person to me" [54].

Sub-theme 2: the right thing Almost all parents described wanting to act in an ethical manner when making decisions, based on moral and religious beliefs. Although the two domains of morality and religion overlapped at times, they appeared to be distinct entities for many parents, with one women referring to the decision being "a moral decision, not a religious one" [65], whilst another felt that her decision "had a lot to do with our religious faith" [47]. Parents described struggling with their conscience and beliefs around termination, with some women believing they would "go to hell" for terminating a pregnancy [58], and struggling with their decision until the process was over, believing that was "the last window of opportunity to stop it from happening" [62]. Some parents described seeking confirmation that their choice was morally acceptable by seeking advice from religious clergy. Often parents received the message that they should do "what they felt was necessary, even if it included termination" [46]; however, others were encouraged to continue with the pregnancy, with the message that "God would only give a person what they could cope with" [72].

Sub-theme 3: learning from experience Parents considered learning from their own past experiences, reporting beliefs about the acceptability of termination which had "originated from past experiences with pregnancy termination" [47], with some stating it had influenced their current decision. Whilst parents' contact with other people diagnosed with the disorder presented them with considerations of "all the good times" [77], they also discussed how they "remember the really hard times" [77], which added further intricacy to their decision-making.

"I already provoked an abortion once before, and now I think that it's a punishment. I swore not to do it again" [64]. 
Table 2 Characteristics of included studies presented as studies exploring experiences of parents who continued pregnancies, terminated pregnancies, and studies exploring both

\begin{tabular}{|c|c|c|c|c|c|c|c|c|}
\hline & Study & Research Aims & $\begin{array}{l}\text { Sample size \& } \\
\text { Diagnosis }\end{array}$ & $\begin{array}{l}\text { Participant } \\
\text { Characteristics }\end{array}$ & Data Collection & $\begin{array}{l}\text { Methodology \& } \\
\text { Analysis }\end{array}$ & Themes & $\begin{array}{l}\text { CASP } \\
\text { Rating } \\
\text { (Cate- } \\
\text { gory) }\end{array}$ \\
\hline \multicolumn{9}{|c|}{ Studies describing pregnancy continuation } \\
\hline 1 & $\begin{array}{l}\text { Helm et al. } \\
\text { [46] } 1998 \\
\text { USA }\end{array}$ & $\begin{array}{l}\text { To explore the } \\
\text { experience of } \\
\text { mothers who } \\
\text { continued a } \\
\text { pregnancy following } \\
\text { diagnosis of Down } \\
\text { Syndrome (DS) }\end{array}$ & $\begin{array}{l}\text { Ten mothers } \\
\text { who had } \\
\text { received a } \\
\text { prenatal } \\
\text { diagnosis of DS }\end{array}$ & $\begin{array}{l}\text { Six Catholic, three } \\
\text { Protestant, one } \\
\text { Jewish } \\
\text { All European- } \\
\text { American } \\
\text { Aged } 28-42 \\
\text { Nine living with } \\
\text { husband, one single }\end{array}$ & $\begin{array}{l}\text { Recruited through a } \\
\text { University affiliated } \\
\text { centre at local } \\
\text { hospitals. Mothers } \\
\text { expressed interest in } \\
\text { the study before } \\
\text { being contacted. } \\
\text { Semi-structured, } \\
\text { open-ended inter- } \\
\text { views were then } \\
\text { conducted over 2-4 } \\
\text { h }\end{array}$ & $\begin{array}{l}\text { Thematic } \\
\text { analysis with } \\
\text { approximately } \\
10 \% \text { of interview } \\
\text { content used }\end{array}$ & $\begin{array}{l}\text { Religion } \\
\text { Morality } \\
\text { Personal } \\
\text { experience } \\
\text { with people } \\
\text { with } \\
\text { disabilities. A } \\
\text { Previous } \\
\text { infertility } \\
\text { Family } \\
\text { Friends } \\
\text { Religious } \\
\text { leaders } \\
\text { Clinicians } \\
\text { Genetic } \\
\text { counsellors }\end{array}$ & $8(A)$ \\
\hline 2 & $\begin{array}{l}\text { Redlinger- } \\
\text { Grosse et al. } \\
\text { [47] } 2002 \\
\text { USA }\end{array}$ & $\begin{array}{l}\text { To explore parents' } \\
\text { experiences in } \\
\text { deciding to continue } \\
\text { a pregnancy, given } \\
\text { the prenatal } \\
\text { diagnosis } \\
\text { Holoprosen-cephaly } \\
\text { (HPE) }\end{array}$ & $\begin{array}{l}\text { Twenty-four } \\
\text { parents who } \\
\text { had received a } \\
\text { prenatal } \\
\text { diagnosis of } \\
\text { HPE }\end{array}$ & $\begin{array}{l}\text { Fourteen women } \\
\text { and } 10 \text { men } \\
\text { Aged } 23-50 \\
\text { Twenty-one white, } 2 \\
\text { Hispanic and } 1 \text { Asian } \\
\text { Twenty-two married, } \\
1 \text { single and } 1 \\
\text { separated or } \\
\text { divorced. } \\
\text { Twenty-three had } \\
\text { living child, } 1 \text { had } \\
\text { no living children } \\
\text { Thirteen Catholic, } 7 \\
\text { Protestant and } 4 \\
\text { Baptist } \\
\text { Twelve completed } \\
\text { high school, } 6 \\
\text { college, } 5 \text { graduate } \\
\text { degree and } 1 \text { post- } \\
\text { graduate degree } \\
\text { Fifteen were } \\
\text { employed full time, } \\
7 \text { unemployed and } 2 \\
\text { worked part-time }\end{array}$ & $\begin{array}{l}\text { Participants were } \\
\text { recruited from a } \\
\text { conference, research } \\
\text { centre, university } \\
\text { and support services } \\
\text { for families affected } \\
\text { by HPE during } 2000 \text {. } \\
\text { In-person interviews } \\
\text { were conducted for } \\
8 \text { parents attending } \\
\text { the conference, with } \\
\text { telephone interviews } \\
\text { for } 16 \text { parents re- } \\
\text { cruited through the } \\
\text { remaining sites, } \\
\text { using open-ended } \\
\text { questions }\end{array}$ & $\begin{array}{l}\text { Thematic } \\
\text { analysis [48] }\end{array}$ & $\begin{array}{l}\text { Religion } \\
\text { Fatalism } \\
\text { Morality } \\
\text { Valuing } \\
\text { mother role } \\
\text { Previous } \\
\text { infertility } \\
\text { Prior } \\
\text { pregnancy } \\
\text { termination } \\
\text { Anticipated } \\
\text { guilt } \\
\text { Personal } \\
\text { connection to } \\
\text { HPE }\end{array}$ & $8(A)$ \\
\hline 3 & $\begin{array}{l}\text { Rempel et al. } \\
\text { [49] } 2004 \\
\text { USA }\end{array}$ & $\begin{array}{l}\text { To explore parents' } \\
\text { decision-making and } \\
\text { interactions with } \\
\text { health care profes- } \\
\text { sionals during the } \\
\text { antenatal diagnosis } \\
\text { of Coronary Heart } \\
\text { Diseases (CHD) }\end{array}$ & $\begin{array}{l}\text { Thirty-four } \\
\text { parents who } \\
\text { had received a } \\
\text { prenatal } \\
\text { diagnosis of } \\
\text { CHD }\end{array}$ & $\begin{array}{l}\text { Nineteen mothers } \\
\text { and } 15 \text { fathers } \\
\text { All intended to } \\
\text { continue with the } \\
\text { pregnancy } \\
\text { Over half first-time } \\
\text { parents } \\
\text { Gestational age } \\
\text { when diagnosed } \\
\text { was 18-36 weeks }\end{array}$ & $\begin{array}{l}\text { In-depth, open- } \\
\text { ended interviews } \\
\text { during the preg- } \\
\text { nancy, } 1 \text { month after } \\
\text { the baby's birth and } \\
\text { between } 4 \text { and } 6 \\
\text { months after birth. } \\
\text { The interviews } \\
\text { ceased following } \\
\text { data saturation }\end{array}$ & $\begin{array}{l}\text { Symbolic } \\
\text { interactionism } \\
\text { guided analysis } \\
{[50]}\end{array}$ & $\begin{array}{l}\text { Search for } \\
\text { information, } \\
\text { Impact on } \\
\text { themselves } \\
\text { Impact on } \\
\text { wider family } \\
\text { Health care } \\
\text { professionals } \\
\text { Attachment }\end{array}$ & $8(A)$ \\
\hline 4 & $\begin{array}{l}\text { Hickerton et } \\
\text { al. [51] } 2012 \\
\text { Australia }\end{array}$ & $\begin{array}{l}\text { To explore parents' } \\
\text { experience of } \\
\text { continuing a } \\
\text { pregnancy where a } \\
\text { genetic condition } \\
\text { was detected } \\
\text { prenatally }\end{array}$ & Nine parents & $\begin{array}{l}\text { Five mothers and } 4 \\
\text { fathers of children < } \\
10 \text { years old with } \\
\text { life-limiting disorders } \\
\text { All had prenatal } \\
\text { diagnosis, or were } \\
\text { warned of high } \\
\text { reproductive risk } \\
\text { Four skeletal } \\
\text { disorders, } 2 \text { other } \\
\text { organ difficulties, } 2\end{array}$ & $\begin{array}{l}\text { Purposive sampling } \\
\text { via advertisement in } \\
\text { the newsletter of a } \\
\text { genetic support } \\
\text { network, or through } \\
\text { receiving a letter } \\
\text { from staff at genetic } \\
\text { clinics } \\
\text { Semi-structured face- } \\
\text { to-face interviews } \\
\text { were conducted }\end{array}$ & $\begin{array}{l}\text { Grounded } \\
\text { Theory using a } \\
\text { constant } \\
\text { comparative } \\
\text { approach [52] }\end{array}$ & $\begin{array}{l}\text { Morality } \\
\text { Quality of life }\end{array}$ & $9(A)$ \\
\hline
\end{tabular}


Table 2 Characteristics of included studies presented as studies exploring experiences of parents who continued pregnancies, terminated pregnancies, and studies exploring both (Continued)

\begin{tabular}{|c|c|c|c|c|c|c|c|c|}
\hline & Study & Research Aims & $\begin{array}{l}\text { Sample size \& } \\
\text { Diagnosis }\end{array}$ & $\begin{array}{l}\text { Participant } \\
\text { Characteristics }\end{array}$ & Data Collection & $\begin{array}{l}\text { Methodology \& } \\
\text { Analysis }\end{array}$ & Themes & $\begin{array}{l}\text { CASP } \\
\text { Rating } \\
\text { (Cate- } \\
\text { gory) }\end{array}$ \\
\hline & & & & $\begin{array}{l}\text { Trisomy syndromes } \\
\text { and } 1 \text { other } \\
\text { chromosomal } \\
\text { disorder } \\
\text { All fluent in English } \\
\text { All married and lived } \\
\text { together }\end{array}$ & $\begin{array}{l}\text { with mothers and fa- } \\
\text { thers separately, ran- } \\
\text { ging from } .5-.2 \mathrm{~h}\end{array}$ & & & \\
\hline 5 & $\begin{array}{l}\text { Hurford et al. } \\
\text { [53] } 2013 \\
\text { USA }\end{array}$ & $\begin{array}{l}\text { To explore women's } \\
\text { decisions to } \\
\text { continue a } \\
\text { pregnancy affected } \\
\text { by DS after prenatal } \\
\text { diagnosis, and the } \\
\text { factors that affected } \\
\text { their decision }\end{array}$ & $\begin{array}{l}\text { Fifty-six } \\
\text { mothers who } \\
\text { had received a } \\
\text { prenatal } \\
\text { diagnosis of DS }\end{array}$ & $\begin{array}{l}\text { Women aged } \\
\text { between } 21 \text { and } 44 \\
\text { Forty-one Caucasian, } \\
8 \text { Hispanic, } 3 \text { Asian, } 2 \\
\text { African American, } \\
\text { and } 1 \text { other, } \\
\text { Fifty Christians, } 2 \\
\text { Atheist, } 1 \text { Muslim, } 1 \\
\text { Jewish, } \\
\text { Forty-four very/ } \\
\text { somewhat religious, } \\
11 \text { not/not very } \\
\text { religious, } \\
\text { Thirty-two had a } \\
\text { college education, } \\
17 \text { postgraduate } \\
\text { qualifications, } 5 \text { high } \\
\text { school education }\end{array}$ & $\begin{array}{l}\text { An information sheet } \\
\text { was sent through } \\
\text { respective } \\
\text { organizations } \\
\text { This information } \\
\text { sheet provided the } \\
\text { web address for the } \\
\text { online survey which } \\
\text { included } 9 \text { free } \\
\text { response questions } \\
\text { analysed for this } \\
\text { study }\end{array}$ & $\begin{array}{l}\text { Thematic } \\
\text { analysis }\end{array}$ & $\begin{array}{l}\text { Attachment } \\
\text { Religion } \\
\text { Morality } \\
\text { Personification } \\
\text { of fetus } \\
\text { Family } \\
\text { Friends } \\
\text { Belief fetus is } \\
\text { alive } \\
\text { Fatalism } \\
\text { Previous } \\
\text { infertility }\end{array}$ & $8(A)$ \\
\hline 6 & $\begin{array}{l}\text { Guon et al. } \\
\text { [54] } 2014 \\
\text { Worldwide }\end{array}$ & $\begin{array}{l}\text { To explore decision- } \\
\text { making of parents } \\
\text { who continued their } \\
\text { pregnancy after a } \\
\text { pre-natal diagnosis } \\
\text { of Trisomy 13-18 }\end{array}$ & $\begin{array}{l}\text { One-hundred } \\
\text { and twenty- } \\
\text { eight parents } \\
\text { who had re- } \\
\text { ceived a pre- } \\
\text { natal diagnosis } \\
\text { of Trisomy 13- } \\
18\end{array}$ & $\begin{array}{l}\text { Thirty men and } 98 \\
\text { women who had } \\
\text { accessed Facebook } \\
\text { and other support } \\
\text { sites dedicated to } \\
\text { Trisomy } 13-18 . \\
\text { One hundred and } \\
\text { six were from the } \\
\text { US, } 6 \text { Canada, } 6 \text { UK, } \\
9 \text { from } 12 \text { other } \\
\text { countries } \\
\text { Parent's median age } \\
\text { was } 38 \\
\text { All completed high } \\
\text { school, } 94 \text { at least } \\
\text { one university } \\
\text { degree, } 27 \text { also } \\
\text { completed } \\
\text { postgraduate studies } \\
\text { One-hundred and } \\
\text { nine were religious, } \\
\text { with } 73 \text { attending } \\
\text { religious services } \\
\text { All were parents of } \\
\text { children who live(d) } \\
\text { with full T13-18, } \\
\text { mosaicism, and } \\
\text { other structural } \\
\text { variations involving } \\
\text { chromosomes } 13 \\
\text { and } 18 \\
\text { Ninety-seven had } \\
\text { full T13 or } 18 \text { The } \\
\text { median age of } \\
\text { survivors was } 3 \text { years }\end{array}$ & $\begin{array}{l}\text { Participants were } \\
\text { recruited on English } \\
\text { speaking, online } \\
\text { support sites relating } \\
\text { to Trisomy 13-18 } \\
\text { respondents } \\
\text { completed a } \\
\text { questionnaire, with } 5 \\
\text { open ended } \\
\text { questions from this } \\
\text { used within the } \\
\text { study analysis }\end{array}$ & $\begin{array}{l}\text { Thematic } \\
\text { qualitative } \\
\text { content analysis }\end{array}$ & $\begin{array}{l}\text { Morality } \\
\text { Religion } \\
\text { Personal values } \\
\text { Attachment } \\
\text { Uncertainty } \\
\text { Hope } \\
\text { Desire to be a } \\
\text { parent } \\
\text { Desire to meet } \\
\text { their child } \\
\text { Pressure from } \\
\text { others }\end{array}$ & $9(A)$ \\
\hline 7 & $\begin{array}{l}\text { Gesser- } \\
\text { Edelburg \& }\end{array}$ & $\begin{array}{l}\text { To explore Muslim } \\
\text { Arab women's }\end{array}$ & $\begin{array}{l}\text { Twenty-four } \\
\text { mothers who }\end{array}$ & $\begin{array}{l}\text { Recruited between } \\
2014 \text { and } 2015\end{array}$ & $\begin{array}{l}\text { Five participants } \\
\text { were recruited by }\end{array}$ & $\begin{array}{l}\text { Constructivist } \\
\text { classical }\end{array}$ & $\begin{array}{l}\text { Religion } \\
\text { Fatalism }\end{array}$ & $9(A)$ \\
\hline
\end{tabular}


Table 2 Characteristics of included studies presented as studies exploring experiences of parents who continued pregnancies, terminated pregnancies, and studies exploring both (Continued)

\begin{tabular}{|c|c|c|c|c|c|c|c|c|}
\hline & Study & Research Aims & $\begin{array}{l}\text { Sample size \& } \\
\text { Diagnosis }\end{array}$ & $\begin{array}{l}\text { Participant } \\
\text { Characteristics }\end{array}$ & Data Collection & $\begin{array}{l}\text { Methodology \& } \\
\text { Analysis }\end{array}$ & Themes & $\begin{array}{l}\text { CASP } \\
\text { Rating } \\
\text { (Cate- } \\
\text { gory) }\end{array}$ \\
\hline & $\begin{array}{l}\text { Shahbari [55] } \\
2017 \text { Israel }\end{array}$ & $\begin{array}{l}\text { reasons for } \\
\text { continuation of a } \\
\text { pregnancy following } \\
\text { the detection of a } \\
\text { congenital anomaly }\end{array}$ & $\begin{array}{l}\text { had received a } \\
\text { diagnosis of } \\
\text { congenital } \\
\text { abnormal-ities }\end{array}$ & $\begin{array}{l}\text { All married } \\
\text { All Muslim } \\
\text { Aged between } 21 \\
\text { and } 39\end{array}$ & $\begin{array}{l}\text { professionals; the } \\
\text { rest were through } \\
\text { snowballing } \\
\text { Interviews lasted 50- } \\
60 \text { min, with } \\
\text { recruitment ceased } \\
\text { following saturation } \\
\text { The questions were } \\
\text { open-ended and } \\
\text { probes were used to } \\
\text { elicit more } \\
\text { information }\end{array}$ & $\begin{array}{l}\text { grounded theory } \\
{[56]}\end{array}$ & $\begin{array}{l}\text { Attachment } \\
\text { Pressure }\end{array}$ & \\
\hline 8 & $\begin{array}{l}\text { Moudi \& } \\
\text { Miri- } \\
\text { Moghaddam } \\
\text { [57] } 2017 \\
\text { Iran }\end{array}$ & $\begin{array}{l}\text { To explore the } \\
\text { reasons women with } \\
\text { a pregnancy } \\
\text { affected by Beta- } \\
\text { Thalassemia (B-TM) } \\
\text { continued with } \\
\text { pregnancy }\end{array}$ & $\begin{array}{l}\text { Thirty-nine } \\
\text { mothers who } \\
\text { had received a } \\
\text { prenatal } \\
\text { diagnosis of } \\
\text { BTM }\end{array}$ & $\begin{array}{l}\text { Women who had } \\
\text { continued with } \\
\text { pregnancy } \\
\text { Thirty-three were of } \\
\text { Sunni religion and } 6 \\
\text { Shiite Muslims } \\
\text { Aged between } 20 \\
\text { and } 34 \text { years } \\
\text { Five of the women } \\
\text { had previously } \\
\text { aborted } 1 \\
\text { pregnancy, whilst } \\
\text { one woman had } \\
\text { aborted } 2 \text { or more } \\
\text { previous } \\
\text { pregnancies }\end{array}$ & $\begin{array}{l}\text { Exploratory } \\
\text { qualitative methods } \\
\text { after purposive } \\
\text { sampling were used } \\
\text { to identify potential } \\
\text { cases In-depth, semi- } \\
\text { structured interviews } \\
\text { were conducted be- } \\
\text { tween } 2012 \text { and } \\
\text { 2013, terminating } \\
\text { when data saturation } \\
\text { was reached Inter- } \\
\text { views lasted approxi- } \\
\text { mately } 1 \mathrm{~h} \text { and used } \\
\text { open-ended } \\
\text { questions }\end{array}$ & $\begin{array}{l}\text { Grounded theory } \\
\text { principles [56] }\end{array}$ & $\begin{array}{l}\text { Belief in } \\
\text { accuracy } \\
\text { Trust in } \\
\text { professionals } \\
\text { Lack of } \\
\text { understanding } \\
\text { Consequences } \\
\text { to family } \\
\text { Family } \\
\text { opinions } \\
\text { Future fertility } \\
\text { Preference for } \\
\text { large family } \\
\text { Preference for } \\
\text { male fetus } \\
\text { Lack of siblings } \\
\text { Hope for } \\
\text { family with } \\
\text { husband } \\
\text { Morality } \\
\text { Religion } \\
\text { Attachment }\end{array}$ & $9(A)$ \\
\hline \multicolumn{9}{|c|}{ Studies describing pregnancy termination } \\
\hline 9 & $\begin{array}{l}\text { Bryar [58] } \\
1997 \text { USA }\end{array}$ & $\begin{array}{l}\text { To explore the } \\
\text { experiences of } \\
\text { women terminating } \\
\text { a pregnancy in the } \\
\text { 2nd trimester due to } \\
\text { discovery of a fetal } \\
\text { abnormality }\end{array}$ & $\begin{array}{l}\text { Three women } \\
\text { who had } \\
\text { received a } \\
\text { diagnosis of } \\
\text { severe fetal } \\
\text { abnormal-ity }\end{array}$ & $\begin{array}{l}\text { Women living in } \\
\text { USA who had } \\
\text { attended a clinic for } \\
\text { abortion } \\
\text { Aged between } 30 \\
\text { and } 40 \text { years } \\
\text { All married, white, } \\
\text { middle class } \\
\text { graduates }\end{array}$ & $\begin{array}{l}\text { Convenience } \\
\text { sampling, } \\
\text { approaching all } \\
\text { women attending } \\
\text { follow-up visit } 4 \\
\text { weeks after their } \\
\text { abortion, within a } \\
\text { single private out- } \\
\text { patient perinatal } \\
\text { practice } \\
\text { Unstructured } \\
\text { interviews were } \\
\text { completed 4-6 } \\
\text { weeks after } \\
\text { termination } \\
\text { The interviews lasted } \\
75-90 \text { min and asked } \\
\text { open questions with } \\
\text { additional questions } \\
\text { asked for clarification }\end{array}$ & $\begin{array}{l}\text { Phenomenology } \\
\text { [59] }\end{array}$ & $\begin{array}{l}\text { Religion } \\
\text { Family impact } \\
\text { Societal } \\
\text { expectations } \\
\text { Impact on self } \\
\text { Potential } \\
\text { future } \\
\text { difficulties } \\
\text { Seriousness of } \\
\text { disorder }\end{array}$ & $9(\mathrm{~A})$ \\
\hline 10 & $\begin{array}{l}\text { Ferreira da } \\
\text { Costa et al. } \\
\text { [60] } 2005 \\
\text { Brazil }\end{array}$ & $\begin{array}{l}\text { To explore the } \\
\text { experience of } \\
\text { women in Brazil } \\
\text { during the process } \\
\text { of abortion for fetal } \\
\text { disorder }\end{array}$ & $\begin{array}{l}\text { Ten women } \\
\text { who had } \\
\text { received a } \\
\text { diagnosis of } \\
\text { severe fetal } \\
\text { disorder }\end{array}$ & $\begin{array}{l}\text { Women who had } \\
\text { terminated a } \\
\text { pregnancy following } \\
\text { legal authorisation in } \\
2002 \\
\text { Aged 17-29 } \\
\text { Two were single }\end{array}$ & $\begin{array}{l}\text { Interviews were } \\
\text { conducted } 40 \text { days } \\
\text { after termination } \\
\text { when the women } \\
\text { returned for post- } \\
\text { termination check- } \\
\text { up and genetic }\end{array}$ & $\begin{array}{l}\text { Thematic } \\
\text { analysis with the } \\
\text { methodology of } \\
\text { Minayo [61] was } \\
\text { used }\end{array}$ & $\begin{array}{l}\text { Seriousness of } \\
\text { disorder } \\
\text { Risk to own } \\
\text { health } \\
\text { Reducing } \\
\text { suffering }\end{array}$ & $9(A)$ \\
\hline
\end{tabular}


Table 2 Characteristics of included studies presented as studies exploring experiences of parents who continued pregnancies, terminated pregnancies, and studies exploring both (Continued)

\begin{tabular}{|c|c|c|c|c|c|c|c|c|}
\hline & Study & Research Aims & $\begin{array}{l}\text { Sample size \& } \\
\text { Diagnosis }\end{array}$ & $\begin{array}{l}\text { Participant } \\
\text { Characteristics }\end{array}$ & Data Collection & $\begin{array}{l}\text { Methodology \& } \\
\text { Analysis }\end{array}$ & Themes & $\begin{array}{l}\text { CASP } \\
\text { Rating } \\
\text { (Cate- } \\
\text { gory) }\end{array}$ \\
\hline & & & & $\begin{array}{l}\text { All had completed } \\
\text { at least primary } \\
\text { school education }\end{array}$ & $\begin{array}{l}\text { counselling } \\
\text { The sample was } \\
\text { determined by } \\
\text { saturation, with } \\
\text { semi-structured in- } \\
\text { terviews following a } \\
\text { thematic script }\end{array}$ & & & \\
\hline 11 & $\begin{array}{l}\text { Leichtentri-tt } \\
\text { [62] } 2011 \\
\text { Israel }\end{array}$ & $\begin{array}{l}\text { To explore the } \\
\text { experience of } \\
\text { women who } \\
\text { undergo feticide in } \\
\text { Israel }\end{array}$ & $\begin{array}{l}\text { Thirteen } \\
\text { women who } \\
\text { had received } \\
\text { positive } \\
\text { prenatal } \\
\text { diagnosis }\end{array}$ & $\begin{array}{l}\text { Aged between } 28 \\
\text { and } 41 \\
\text { All married, with one } \\
\text { woman remarried } \\
\text { Five received a } \\
\text { prenatal diagnosis of } \\
\text { Brain or (CNS) } \\
\text { defects, } 3 \text { received } \\
\text { diagnosis relating to } \\
\text { other organ } \\
\text { difficulties, } 2 \text { heart } \\
\text { defects, } 1 \text { sex } \\
\text { chromosome } \\
\text { abnormalities, } 1 \\
\text { other chromosome } \\
\text { abnormalities and } 1 \\
\text { fetal growth } \\
\text { restriction }\end{array}$ & $\begin{array}{l}\text { The women were } \\
\text { recruited from one } \\
\text { of the largest } \\
\text { hospitals in Israel, } \\
\text { and through } \\
\text { snowballing } \\
\text { techniques between } \\
2008 \text { and } 09 \\
\text { Interviews took place } \\
\text { 4-24 months after } \\
\text { the termination } \\
\text { using in-depth semi- } \\
\text { structured interviews } \\
\text { lasting approxi- } \\
\text { mately } 3 \text { h }\end{array}$ & $\begin{array}{l}\text { Thematic } \\
\text { analysis using } \\
\text { the approach of } \\
\text { Gubrium and } \\
\text { Holstein [63] }\end{array}$ & $\begin{array}{l}\text { Pressure from } \\
\text { professionals } \\
\text { Uncertainty } \\
\text { Preventing } \\
\text { suffering } \\
\text { Time pressure } \\
\text { Legal } \\
\text { requirements } \\
\text { Family opinion }\end{array}$ & $9(A)$ \\
\hline 12 & $\begin{array}{l}\text { Benute et al. } \\
\text { [64] } 2012 \\
\text { Brazil }\end{array}$ & $\begin{array}{l}\text { To explore women's } \\
\text { reasons for } \\
\text { termination of } \\
\text { pregnancies after } \\
\text { ultrasound } \\
\text { presented findings } \\
\text { consistent with } \\
\text { lethal fetal } \\
\text { malformation }\end{array}$ & $\begin{array}{l}\text { Two-hundred } \\
\text { and forty-nine } \\
\text { women who re- } \\
\text { ceived positive } \\
\text { prenatal results }\end{array}$ & $\begin{array}{l}\text { Women recruited } \\
\text { between } 1998 \text { and } \\
2008 \\
\text { Aged between } 18 \\
\text { and } 33 \text { years } \\
\text { One-hundred and } \\
\text { thirty-five received } \\
\text { diagnosis of anen- } \\
\text { cephaly, } 26 \text { urinary } \\
\text { disorders, } 24 \text { genetic } \\
\text { syndromes, } 21 \text { CNS, } \\
21 \text { multiple MF, 13 } \\
\text { Column MF, } 8 \text { con- } \\
\text { joined twins and } 1 \\
\text { abdominal wall } \\
\text { disorder } \\
\text { Fetus death prior to } \\
\text { the interview led to } \\
\text { exclusion } \\
\text { One-hundred and } \\
\text { sixty-two were Cath- } \\
\text { olic, } 54 \text { Evangelical } \\
\text { One-hundred and } \\
\text { seventy-seven co- } \\
\text { habited with partner, } \\
72 \text { single } \\
\text { One-hundred and } \\
\text { seventy-two opted } \\
\text { for termination, } 77 \\
\text { had not requested } \\
\text { abortion }\end{array}$ & $\begin{array}{l}\text { The sampling was } \\
\text { carried out by } \\
\text { exhaustion. A } \\
\text { psychologist } \\
\text { conducted open } \\
\text { interviews } \\
\text { immediately after } \\
\text { the ultrasound } \\
\text { diagnosis, with each } \\
\text { interview lasting } \\
\text { approximately } 1 \mathrm{~h}\end{array}$ & $\begin{array}{l}\text { Content Analysis } \\
\text { was utilised }\end{array}$ & $\begin{array}{l}\text { Reducing } \\
\text { suffering } \\
\text { Guilt } \\
\text { Morality }\end{array}$ & $8(A)$ \\
\hline 13 & $\begin{array}{l}\text { Gawron et al. } \\
\text { [65] } 2015 \\
\text { USA }\end{array}$ & $\begin{array}{l}\text { To explore the } \\
\text { reasons for } \\
\text { termination timing } \\
\text { among patients } \\
\text { whose pregnancy } \\
\text { was effected by fetal }\end{array}$ & $\begin{array}{l}\text { Thirty women } \\
\text { who had } \\
\text { received } \\
\text { diagnosis of } \\
\text { severe fetal } \\
\text { disorders }\end{array}$ & $\begin{array}{l}\text { English speaking, } \\
\text { adult women } \\
\text { presenting for } \\
\text { pregnancy } \\
\text { termination between } \\
12 \text { and } 24 \text { weeks at a }\end{array}$ & $\begin{array}{l}\text { Convenience } \\
\text { sampling with } \\
\text { phone or pre- } \\
\text { operative consult- } \\
\text { ation recruitment } \\
\text { until data saturation }\end{array}$ & $\begin{array}{l}\text { Interviews were } \\
\text { analysed using } \\
\text { latent grounded } \\
\text { theory [56] }\end{array}$ & $\begin{array}{l}\text { Partner } \\
\text { Shared } \\
\text { decision- } \\
\text { making } \\
\text { Family } \\
\text { Friends }\end{array}$ & $8(A)$ \\
\hline
\end{tabular}


Table 2 Characteristics of included studies presented as studies exploring experiences of parents who continued pregnancies, terminated pregnancies, and studies exploring both (Continued)

\begin{tabular}{|c|c|c|c|c|c|c|c|}
\hline Study & Research Aims & $\begin{array}{l}\text { Sample size \& } \\
\text { Diagnosis }\end{array}$ & $\begin{array}{l}\text { Participant } \\
\text { Characteristics }\end{array}$ & Data Collection & $\begin{array}{l}\text { Methodology \& } \\
\text { Analysis }\end{array}$ & Themes & $\begin{array}{l}\text { CASP } \\
\text { Rating } \\
\text { (Cate-- } \\
\text { gory) }\end{array}$ \\
\hline & abnormalities & & $\begin{array}{l}\text { tertiary referral family } \\
\text { planning clinic } \\
\text { between } 2011 \text { and } \\
12 \\
\text { Aged between } 26 \\
\text { and } 44 \\
\text { All were married or } \\
\text { in a relationship } \\
\text { All had some } \\
\text { college education, } \\
12 \text { post-college } \\
\text { qualifications } \\
\text { Twenty were } \\
\text { employed } \\
\text { Sixteen were } \\
\text { Christian, } 9 \text { Athiest, } 3 \\
\text { Jewish, } 1 \text { Muslim } \\
\text { and } 1 \text { Hindi } \\
\text { Several women had } \\
\text { previously } \\
\text { terminated } \\
\text { pregnancies, } \\
\text { including } 3 \text { for fetal } \\
\text { abnormalities }\end{array}$ & $\begin{array}{l}\text { reached } \\
\text { The interview } \\
\text { occurred on the first } \\
\text { of a 2-day termin- } \\
\text { ation process, prior } \\
\text { to medical team } \\
\text { interaction } \\
\text { Semi-structured } \\
\text { interviews using } \\
\text { guide focused on } \\
\text { decision-making was } \\
\text { used, with follow-up } \\
\text { questions lasting up } \\
\text { to } 60 \text { min }\end{array}$ & & $\begin{array}{l}\text { Religion } \\
\text { Suffering for } \\
\text { fetus }\end{array}$ & \\
\hline $\begin{array}{l}14 \text { loannou et } \\
\text { al. [66] } 2015 \\
\text { Australia }\end{array}$ & $\begin{array}{l}\text { To explore the } \\
\text { experiences of } \\
\text { couples that were } \\
\text { both identified as } \\
\text { carriers of Cystic } \\
\text { Fibrosis (CF), and the } \\
\text { reproductive } \\
\text { decision-making and } \\
\text { psychosocial impact } \\
\text { when pregnant }\end{array}$ & $\begin{array}{l}\text { Four parents } \\
\text { who had } \\
\text { received a } \\
\text { prenatal } \\
\text { diagnosis of CF }\end{array}$ & $\begin{array}{l}\text { Four Individuals } \\
\text { forming } 2 \text { couples } \\
\text { taken from a larger } \\
\text { sample included } \\
\text { within the study } \\
\text { were screened } \\
\text { between } 2006 \text { and } \\
2012 \text { and found to } \\
\text { be in a 'carrier } \\
\text { couple' } \\
\text { One of the } \\
\text { pregnancies had } \\
\text { occurred through } \\
\text { IVF } \\
\text { Both pregnancies } \\
\text { were terminated }\end{array}$ & $\begin{array}{l}\text { Open-ended } \\
\text { questions, informed } \\
\text { by the literature and } \\
\text { process of screening, } \\
\text { were used in the } \\
\text { semi-structured } \\
\text { interview schedule }\end{array}$ & $\begin{array}{l}\text { Inductive } \\
\text { content analysis } \\
{[67]}\end{array}$ & $\begin{array}{l}\text { Disbelief } \\
\text { Hope } \\
\text { Prior decision- } \\
\text { making }\end{array}$ & $9(A)$ \\
\hline \multicolumn{8}{|c|}{ Studies describing pregnancy termination and continuation } \\
\hline $\begin{array}{l}15 \text { Sandelows-ki } \\
\text { \& Jones [68] } \\
1996 \text { USA }\end{array}$ & $\begin{array}{l}\text { To explore the } \\
\text { experience of } \\
\text { couples who learn } \\
\text { during pregnancy } \\
\text { that their baby has a } \\
\text { severe fetal } \\
\text { impairment }\end{array}$ & $\begin{array}{l}\text { Twenty-seven } \\
\text { parents who } \\
\text { had been } \\
\text { prenatally } \\
\text { diagnosed with } \\
\text { a severe fetal } \\
\text { disorder }\end{array}$ & $\begin{array}{l}\text { Fifteen women and } \\
12 \text { of their male } \\
\text { partners ( } 11 \text { of } \\
\text { whom were married } \\
\text { couples) } \\
\text { Women aged } \\
\text { between } 19 \text { and } 40 \text {, } \\
\text { men between } 22 \\
\text { and } 39 \\
\text { Largely Euro- } \\
\text { American, except for } \\
1 \text { African-American } \\
\text { couple, } 1 \text { African- } \\
\text { American woman, } \\
\text { and } 1 \text { Asian- } \\
\text { American woman } \\
\text { Two had terminated } \\
\text { previous } \\
\text { pregnancies for } \\
\text { reasons other than } \\
\text { fetal health }\end{array}$ & $\begin{array}{l}\text { Forty interviews } \\
\text { completed with } 10 \\
\text { couples and } 2 \\
\text { women who were } \\
\text { interviewed 2-5 } \\
\text { times, with } 2 \\
\text { couples and } 1 \\
\text { woman interviewed } \\
\text { once } \\
\text { All participants were } \\
\text { interviewed within } \\
11-60 \text { days of } \\
\text { learning of the fetal } \\
\text { impairment or } \\
\text { termination } \\
\text { Five couples } \\
\text { terminating } \\
\text { pregnancies were } \\
\text { subsequently } \\
\text { interviewed around } \\
\text { their due date and }\end{array}$ & $\begin{array}{l}\text { Narrative analysis } \\
\text { [69] }\end{array}$ & $\begin{array}{l}\text { Reducing own } \\
\text { distress } \\
\text { Reducing } \\
\text { fetus' distress } \\
\text { Religion } \\
\text { Attachment } \\
\text { Own needs } \\
\text { Future needs } \\
\text { of fetus } \\
\text { Ability to care } \\
\text { for child with } \\
\text { disability }\end{array}$ & $8(A)$ \\
\hline
\end{tabular}


Table 2 Characteristics of included studies presented as studies exploring experiences of parents who continued pregnancies, terminated pregnancies, and studies exploring both (Continued)

\begin{tabular}{|c|c|c|c|c|c|c|c|c|}
\hline & Study & Research Aims & $\begin{array}{l}\text { Sample size \& } \\
\text { Diagnosis }\end{array}$ & $\begin{array}{l}\text { Participant } \\
\text { Characteristics }\end{array}$ & Data Collection & $\begin{array}{l}\text { Methodology \& } \\
\text { Analysis }\end{array}$ & Themes & $\begin{array}{l}\text { CASP } \\
\text { Rating } \\
\text { (Cate- } \\
\text { gory) }\end{array}$ \\
\hline & & & & $\begin{array}{l}\text { Eight women } \\
\text { continued their } \\
\text { pregnancies, } 2 \text { losing } \\
\text { their babies within } 1 \\
\mathrm{~h} \text { and } 1 \text { week of } \\
\text { birth, } 5 \text { women } \\
\text { terminated } \\
\text { pregnancies with } \\
\text { live fetuses and } 2 \\
\text { women terminated } \\
\text { pregnancies after a } \\
\text { fetal demise }\end{array}$ & $\begin{array}{l}\text { the anniversary date } \\
\text { of termination } \\
\text { Couples continuing } \\
\text { pregnancies were } \\
\text { interviewed 1-2 } \\
\text { times during } \\
\text { pregnancy and 1-4 } \\
\text { times after delivery } \\
\text { Two women } \\
\text { conceived during } \\
\text { the study and they } \\
\text { were interviewed } \\
\text { during a second } \\
\text { round of prenatal } \\
\text { screening Interviews } \\
\text { were conducted in a } \\
\text { minimally structured } \\
\text { manner, with } \\
\text { questions asked only } \\
\text { to clarify }\end{array}$ & & & \\
\hline 16 & $\begin{array}{l}\text { Locock, \& } \\
\text { Alexander } \\
\text { [70] } 2006 \text { UK }\end{array}$ & $\begin{array}{l}\text { To explore how men } \\
\text { experience fetal } \\
\text { screening and } \\
\text { diagnosis }\end{array}$ & $\begin{array}{l}\text { Seventeen } \\
\text { parents were } \\
\text { recruited } \\
\text { following } \\
\text { positive } \\
\text { prenatal } \\
\text { screening }\end{array}$ & $\begin{array}{l}\text { Eight had received } \\
\text { prenatal diagnosis of } \\
\text { chromosomal } \\
\text { defects, } 5 \text { heart } \\
\text { defects, and } 4 \text { neural } \\
\text { tube defects } \\
\text { Parents were } \\
\text { interviewed either } \\
\text { during pregnancy or } \\
\text { within } 2 \text { years after } \\
\text { birth or termination } \\
\text { between } 2003 \text { and } \\
2004\end{array}$ & $\begin{array}{l}\text { Participants were } \\
\text { recruited using a } \\
\text { maximum variation } \\
\text { sample, through a } \\
\text { national network of } \\
\text { GPs, antenatal clinics } \\
\text { and classes, national } \\
\text { voluntary } \\
\text { associations and } \\
\text { support groups } \\
\text { Narrative interviews } \\
\text { were conducted }\end{array}$ & $\begin{array}{l}\text { Modified } \\
\text { grounded theory } \\
\text { approach [71] }\end{array}$ & $\begin{array}{l}\text { Legality } \\
\text { Exclusion } \\
\text { Joint decision- } \\
\text { making } \\
\text { Role as } \\
\text { supporter } \\
\text { Focussing on } \\
\text { positives } \\
\text { Seeking } \\
\text { information } \\
\text { Hard facts } \\
\text { Intuition }\end{array}$ & $9(\mathrm{~A})$ \\
\hline 17 & $\begin{array}{l}\text { Balkan et al. } \\
\text { [41] } 2010 \\
\text { Turkey }\end{array}$ & $\begin{array}{l}\text { To explore factors } \\
\text { influencing parental } \\
\text { decisions to } \\
\text { terminate or } \\
\text { continue a } \\
\text { pregnancy with } \\
\text { chromosomal } \\
\text { abnormality } \\
\text { diagnosed prenatally }\end{array}$ & $\begin{array}{l}\text { Seventy-six } \\
\text { parents were } \\
\text { recruited } \\
\text { following } \\
\text { positive } \\
\text { prenatal } \\
\text { screening }\end{array}$ & $\begin{array}{l}\text { Thirty-eight couples } \\
\text { from South-East } \\
\text { Turkey who received } \\
\text { positive prenatal } \\
\text { diagnosis between } \\
2004 \text { and } 07 \\
\text { All were Muslim } \\
\text { Thirty-two of the } \\
\text { forty receiving a } \\
\text { diagnosis of DS } \\
\text { terminated, all } \\
\text { receiving Trisomy } 18 \\
\text { diagnosis } \\
\text { terminated, and } 8 \text { of } \\
\text { the } 10 \text { receiving a } \\
\text { diagnosis of Turner } \\
\text { Syndrome (TS) } \\
\text { terminated. None of } \\
\text { the } 6 \text { parents } \\
\text { receiving a diagnosis } \\
\text { of Klinfelter } \\
\text { Syndrome (KS) } \\
\text { terminated, with all } \\
4 \text { parents receiving a } \\
\text { diagnosis of } 47 X X X \text {, } \\
2 \text { receiving marker } \\
\text { chromosome } \\
\text { abnormality or } 4 \\
\text { receiving Trisomy } 13 \\
\text { terminating }\end{array}$ & $\begin{array}{l}\text { Semi structured, } \\
\text { face-to-face inter- } \\
\text { views lasting one to } \\
\text { several hours }\end{array}$ & $\begin{array}{l}\text { No explanation } \\
\text { of exact } \\
\text { methodology } \\
\text { However, } \\
\text { authors report } \\
\text { that qualitative } \\
\text { analyses were } \\
\text { conducted }\end{array}$ & $\begin{array}{l}\text { Religion } \\
\text { Seriousness of } \\
\text { condition }\end{array}$ & $6(\mathrm{~B})$ \\
\hline
\end{tabular}


Table 2 Characteristics of included studies presented as studies exploring experiences of parents who continued pregnancies, terminated pregnancies, and studies exploring both (Continued)

\begin{tabular}{|c|c|c|c|c|c|c|c|c|}
\hline & Study & Research Aims & $\begin{array}{l}\text { Sample size \& } \\
\text { Diagnosis }\end{array}$ & $\begin{array}{l}\text { Participant } \\
\text { Characteristics }\end{array}$ & Data Collection & $\begin{array}{l}\text { Methodology \& } \\
\text { Analysis }\end{array}$ & Themes & $\begin{array}{l}\text { CASP } \\
\text { Rating } \\
\text { (Cate- } \\
\text { gory) }\end{array}$ \\
\hline 18 & $\begin{array}{l}\text { France et al. } \\
\text { [72] } 2012 \text { UK }\end{array}$ & $\begin{array}{l}\text { To explore the role } \\
\text { of women's and } \\
\text { couple's experiences } \\
\text { of disabilities in } \\
\text { influencing their } \\
\text { decision regarding } \\
\text { termination }\end{array}$ & $\begin{array}{l}\text { Twenty-eight } \\
\text { parents, who } \\
\text { had received } \\
\text { positive } \\
\text { prenatal } \\
\text { diagnosis }\end{array}$ & $\begin{array}{l}\text { Twenty-four women } \\
\text { and } 4 \text { of their male } \\
\text { partners with prior } \\
\text { experience of a } \\
\text { disability } \\
\text { Ten had received a } \\
\text { prenatal diagnosis of } \\
\text { autosomal disorders, } \\
3 \text { structural } \\
\text { disorders, } 3 \text { heart } \\
\text { defects, } 5 \text { blood } \\
\text { disorders, } 3 \text { multiple } \\
\text { disorders and } 1 \\
\text { muscular disorder } \\
\text { Twenty had } \\
\text { terminated and } 8 \\
\text { continued with their } \\
\text { pregnancy } \\
\text { Interviews } \\
\text { completed } 1 \text {-12 } \\
\text { years after } \\
\text { pregnancy } \\
\text { Participants aged } \\
23-52 \\
\text { One participant was } \\
\text { Pakistani, } 1 \text { Black } \\
\text { Sierra Leone, } 3 \text { Black } \\
\text { Nigerian and } 23 \\
\text { White } \\
\text { Two were single, } 3 \\
\text { cohabiting and } 23 \\
\text { married } \\
\text { People's experiential } \\
\text { knowledge of } \\
\text { disabilitity included } \\
\text { having a disorder } \\
\text { themselves, living } \\
\text { with a disabled } \\
\text { sibling, and talking } \\
\text { to or observing } \\
\text { family, friends, } \\
\text { acquaintances or } \\
\text { cients who had a } \\
\text { disabilive }\end{array}$ & $\begin{array}{l}\text { Data was collected } \\
\text { as part of larger } \\
\text { scale study } \\
\text { Interviews were } \\
\text { originally conducted } \\
\text { by two researchers } \\
\text { between } 2004 \text { and } \\
06 \text { Narrative } \\
\text { interviews with } \\
\text { participants from a } \\
\text { purposive sample } \\
\text { Recruitment via GPs, } \\
\text { hospital consultants, } \\
\text { nurses, support } \\
\text { groups and word of } \\
\text { mouth Interviews } \\
\text { lasted } 1-3 \text { h }\end{array}$ & $\begin{array}{l}\text { Framework } \\
\text { analysis } \\
\text { influenced by } \\
\text { Bury's [73] and } \\
\text { Lawson \& } \\
\text { Pierson's [74] } \\
\text { frameworks }\end{array}$ & $\begin{array}{l}\text { Shared } \\
\text { decisions with } \\
\text { partner } \\
\text { Other's } \\
\text { experiences of } \\
\text { condition } \\
\text { Experiential } \\
\text { knowledge } \\
\text { Their own } \\
\text { suffering } \\
\text { Suffering of } \\
\text { fetus } \\
\text { Religion } \\
\text { Medical advice } \\
\text { Imagined } \\
\text { futures }\end{array}$ & $9(A)$ \\
\hline 19 & $\begin{array}{l}\text { Huyard [43] } \\
2012 \\
\text { Belgium, } \\
\text { France \& } \\
\text { Germany }\end{array}$ & $\begin{array}{l}\text { To explore the } \\
\text { information parents, } \\
\text { whose child has an } \\
\text { intellectual disability, } \\
\text { considered } \\
\text { important when } \\
\text { deciding whether to } \\
\text { terminate following } \\
\text { prenatal diagnosis }\end{array}$ & $\begin{array}{l}\text { Four participa- } \\
\text { nts from a lar- } \\
\text { ger presented } \\
\text { sample, who } \\
\text { had received a } \\
\text { prenatal diag- } \\
\text { nosis of a se- } \\
\text { vere fetal } \\
\text { disorder }\end{array}$ & $\begin{array}{l}\text { Thirty-three } \\
\text { interviews were } \\
\text { conducted in } \\
\text { Germany, France, } \\
\text { and Belgium } \\
\text { between } 2008 \text { and } \\
10 \text { among women, } \\
\text { men, or couples } \\
\text { who had at least } \\
\text { one child with a life- } \\
\text { limiting condition } \\
\text { Only } 4 \text { participants } \\
\text { from this sample } \\
\text { had experienced a } \\
\text { prenatal diagnosis } \\
\text { Of the relevant } 4 \\
\text { parents, } 3 \text { had } \\
\text { continued with the }\end{array}$ & $\begin{array}{l}\text { The interviewees } \\
\text { were recruited } \\
\text { through self-help } \\
\text { groups of parents } \\
\text { whose children have } \\
\text { an intellectual dis- } \\
\text { ability, or through } \\
\text { professionals work- } \\
\text { ing in schools or } \\
\text { residential centres } \\
\text { for people with intel- } \\
\text { lectual disability } \\
\text { Semi-structured in- } \\
\text { terviews were con- } \\
\text { ducted sequentially, } \\
\text { and followed a 30- } \\
\text { item guide }\end{array}$ & $\begin{array}{l}\text { Classical } \\
\text { grounded theory } \\
\text { methods [56] }\end{array}$ & $\begin{array}{l}\text { Longing for } \\
\text { child } \\
\text { Fatalism } \\
\text { Ability to cope } \\
\text { Morality }\end{array}$ & $6(B)$ \\
\hline
\end{tabular}


Table 2 Characteristics of included studies presented as studies exploring experiences of parents who continued pregnancies, terminated pregnancies, and studies exploring both (Continued)

\begin{tabular}{|c|c|c|c|c|c|c|c|c|}
\hline & Study & Research Aims & $\begin{array}{l}\text { Sample size \& } \\
\text { Diagnosis }\end{array}$ & $\begin{array}{l}\text { Participant } \\
\text { Characteristics }\end{array}$ & Data Collection & $\begin{array}{l}\text { Methodology \& } \\
\text { Analysis }\end{array}$ & Themes & $\begin{array}{l}\text { CASP } \\
\text { Rating } \\
\text { (Cate- } \\
\text { gory) } \\
\end{array}$ \\
\hline & & & & $\begin{array}{l}\text { pregnancy, and } 1 \\
\text { had terminated }\end{array}$ & & & & \\
\hline 20 & $\begin{array}{l}\text { Hodgson et } \\
\text { al. [75] } 2016 \\
\text { Australia }\end{array}$ & $\begin{array}{l}\text { To explore social } \\
\text { and professional } \\
\text { supports utilised by } \\
\text { parents at the time } \\
\text { of lethal, life-limiting, } \\
\text { or severely debilitat- } \\
\text { ing fetal diagnosis }\end{array}$ & $\begin{array}{l}\text { One-hundred } \\
\text { and two } \\
\text { parents who } \\
\text { had received a } \\
\text { severe fetal } \\
\text { diagnosis }\end{array}$ & $\begin{array}{l}\text { Seventy-five women } \\
\text { and } 27 \text { men, all } \\
\text { were English } \\
\text { speaking }\end{array}$ & $\begin{array}{l}\text { Purposive, } \\
\text { convenience } \\
\text { sampling was used } \\
\text { Interviews were } \\
\text { conducted with } \\
\text { couples using a } \\
\text { semi-structured } \\
\text { guide following a } \\
\text { narrative and } \\
\text { chronological style }\end{array}$ & $\begin{array}{l}\text { Thematic } \\
\text { analysis }\end{array}$ & $\begin{array}{l}\text { Provision of } \\
\text { information } \\
\text { Attitude of } \\
\text { information } \\
\text { giver } \\
\text { Likely } \\
\text { prognosis } \\
\text { Termination } \\
\text { options } \\
\text { Previous views } \\
\text { Perceived } \\
\text { impact on } \\
\text { family } \\
\text { Other's } \\
\text { perceptions } \\
\text { Other's } \\
\text { experiences }\end{array}$ & $9(\mathrm{~A})$ \\
\hline 21 & $\begin{array}{l}\text { Fleming et al. } \\
\text { [42] } 2016 \\
\text { Switzerland }\end{array}$ & $\begin{array}{l}\text { To explore the } \\
\text { experiences of } \\
\text { parents following } \\
\text { severe fetal } \\
\text { diagnosis }\end{array}$ & $\begin{array}{l}\text { Thirty-two } \\
\text { parents who } \\
\text { had received a } \\
\text { severe prenatal } \\
\text { diagnosis }\end{array}$ & $\begin{array}{l}\text { Seventeen mothers, } \\
1 \text { father and } 7 \\
\text { couples were } \\
\text { recruited between } \\
2013 \text { and } 14 \text { from } \\
\text { the German } \\
\text { speaking part of } \\
\text { Switzerland } \\
\text { All had experienced } \\
\text { a lethal fetal } \\
\text { diagnosis in the } \\
\text { previous } 5 \text { years }\end{array}$ & $\begin{array}{l}\text { Participants were } \\
\text { recruited through a } \\
\text { telephone } \\
\text { counselling service } \\
\text { available to anyone } \\
\text { with interest in } \\
\text { perinatal loss Data } \\
\text { was collected by } \\
\text { semi-structured in- } \\
\text { terviews lasting 40- } \\
90 \text { min }\end{array}$ & $\begin{array}{l}\text { Thematic } \\
\text { analysis in } \\
\text { accordance with } \\
\text { the method of } \\
\text { Braun and Clarke } \\
\text { [76] was utilised }\end{array}$ & $\begin{array}{l}\text { Time pressure } \\
\text { Searching for } \\
\text { information } \\
\text { Financial } \\
\text { implications }\end{array}$ & $6(\mathrm{~B})$ \\
\hline 22 & $\begin{array}{l}\text { Reed \& } \\
\text { Berrier [77] } \\
\text { (2017) USA }\end{array}$ & $\begin{array}{l}\text { To explore decision- } \\
\text { making following } \\
\text { prenatal diagnosis of } \\
\text { DS }\end{array}$ & $\begin{array}{l}\text { Nine parents } \\
\text { who had } \\
\text { received a } \\
\text { diagnosis of DS }\end{array}$ & $\begin{array}{l}\text { Two couples and } 5 \\
\text { mothers recruited } \\
\text { between } 2012 \text { and } \\
2013 \\
\text { All had received a } \\
\text { prenatal diagnosis } \\
\text { within three years } \\
\text { prior to the } \\
\text { interview } \\
\text { Four had continued } \\
\text { their pregnancy, } 3 \\
\text { had terminated and } \\
2 \text { had continued } \\
\text { with an adoption } \\
\text { plan } \\
\text { Aged between } 26 \\
\text { and } 52 \\
\text { Seven females and } 2 \\
\text { males } \\
\text { Seven Caucasians, } 1 \\
\text { Hispanic, and } 1 \text { Bi- } \\
\text { racial participant } \\
\text { Four participants did } \\
\text { not affiliate to any } \\
\text { religion, } 2 \text { were } \\
\text { Catholic, } 2 \\
\text { Protestant and } 1 \\
\text { Lutheran }\end{array}$ & $\begin{array}{l}\text { A convenience } \\
\text { sample was used to } \\
\text { recruit participants, } \\
\text { with expansive } \\
\text { cognitive } \\
\text { interviewing utilised } \\
\text { Interviews were } 90 \\
\text { min in length and } \\
\text { directed participants } \\
\text { to read and } \\
\text { complete the } \\
\text { questionnaire by } \\
\text { "thinking aloud" } \\
\text { whilst "concurrent } \\
\text { probing" } \\
\text { encouraged } \\
\text { participants to } \\
\text { further elaborate }\end{array}$ & $\begin{array}{l}\text { Thematic } \\
\text { analysis [78] }\end{array}$ & $\begin{array}{l}\text { Pressure from } \\
\text { professionals } \\
\text { Provision of } \\
\text { information } \\
\text { Scientific } \\
\text { information } \\
\text { Confidence in } \\
\text { professionals } \\
\text { Ability to care } \\
\text { for child } \\
\text { Family impact } \\
\text { Support } \\
\text { groups } \\
\text { Prior } \\
\text { experience } \\
\text { with DS } \\
\text { Other's } \\
\text { experiences } \\
\text { Age } \\
\text { Attachment } \\
\text { Personal values } \\
\text { Anticipated } \\
\text { quality of life }\end{array}$ & $8(A)$ \\
\hline
\end{tabular}




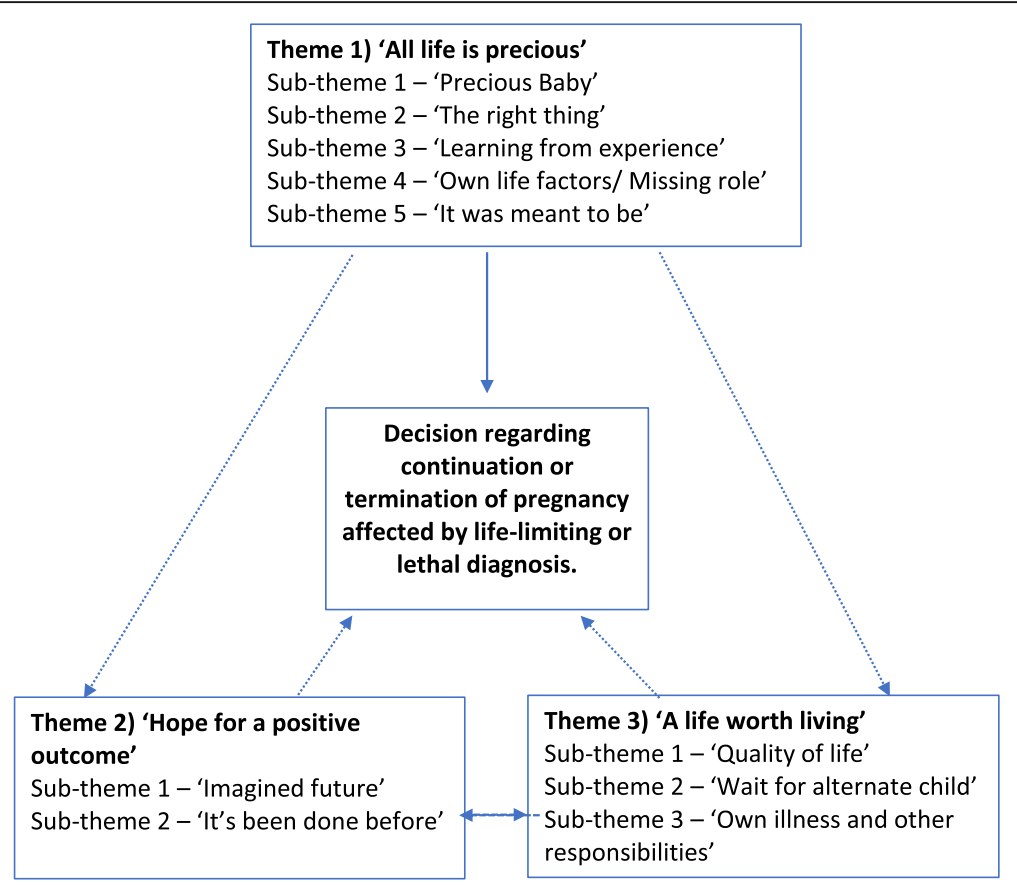

Fig. 2 Diagrammatic representation of decision-making following lethal, life-limiting, or severely debilitating diagnosis

Sub-theme 4: own life factors Parents discussed factors within their life circumstances which had influenced their choices around continuing or terminating an affected pregnancy. Parents highlighted prior attempts of becoming pregnant and suffering miscarriages or fertility difficulties, explaining that they had "tried so hard to have children" [47] and could not consider terminating the pregnancy. Some parents perceived that this pregnancy might be their last chance to have a child due to their advancing age, and felt that they had "waited long enough" [65] to conceive. However, other parents viewed increased age as a concern for their ability to care for an adult with life-limiting disorders when they themselves were older, with fears of "burdening other children or family with this child's care after they died" [65].

Sub-theme 5: it was meant to be Parents described a fatalistic attitude when deciding whether to continue or terminate pregnancies affected by lethal, life-limiting, or severely debilitating disorders, "believing that there was a reason or purpose for being pregnant with a child with Down syndrome" [53]. They explained that they believed there would be a higher purpose for any life, that they were fated to parent the affected child and that it was "meant to be" [54], placing value on the child they had been given, and accepting the outcome of the life regardless of difficulties.

"We were going to let Alaina determine the outcome of her life, no intervention in one way or the other" [54].

\section{Theme 2: Hope for a positive outcome}

The second theme identified was one of hope for a positive outcome. Parents described their hopes for the future, with a healthy or happy child. Parents explained how these hopes influenced their decision on continuing or terminating a pregnancy, based on whether they believed their imagined future was acceptable to them (sub-theme 1). Parents also described how their own and others' belief in their ability to care for a child with lethal, life-limiting, or severely debilitating conditions influenced their decision to continue or terminate a pregnancy. Parents discussed how knowledge of other people's experiences of parenting a child with similar disorders influenced their decision (sub-theme 2).

Sub-theme 1: imagined future Parents explained how their hope that things would be manageable influenced their decision-making. They hoped that their child would survive, and that they and their wider family "would be able to spend some time" with their child [54]. Many parents hoped that all involved might experience some positivity from a traumatic situation.

Often parents did not have faith in the diagnostic certainty and questioned whether "aborting it or terminating it was really necessary" [66]. In these cases, they chose to conduct further research of their own, with the hope of finding positive outcomes for the disorders their unborn child had been diagnosed with or "verifying information from medical providers" [54]. Parents described finding "hope and comfort" [64] through information challenging 
the opinion of professionals, and many described requiring a "more trusted information source" [65] than professional opinion, instead seeking out "online support groups or blogs" [65] to further understand the disorder and make a decision.

"It was difficult, but you dust yourself off, you go home, you read up your books, you read the Internet, you know. And I think you're able to then make informed choices." [70]

Sub-theme 2: It's been done before The knowledge that other people had experienced similar situations as themselves was viewed as beneficial for decision-making. Seeking out "other people's experiences" [72] and learning that other people valued their child, despite disabilities, with some hoping to "adopt a child with Down syndrome" [46], influenced some parents' decisions to continue with a pregnancy. However, others were presented with facts from other parents within similar experiences, regarding the "really hard times that they had" [77] and encouraged to make a fully informed decision.

Parents' beliefs in their own ability to "raise a child with Down syndrome" [77] or their own ability "to cope" [43] with the parental role influenced their decisionmaking. Many parents also relied on the belief others held about their ability to parent a child with specific difficulties, and found this influential in their decisionmaking process.

Advice given by others about different strains or severity of disorder influenced parental beliefs. Parents reported that children with the same condition, but a milder strain, were "going to school; it seems like they have no problems" [57]. Whilst parents were also reported to have harboured incorrect assumptions that "if the baby is diagnosed with thalassemia major in the first pregnancy, it will not re-occur in the second pregnancy" [57], leading to doubt in the accuracy of results and a choice to continue with their pregnancy.

One mother reported that when she called her sister with the news, her response was "You'd make a great mother for a kid with Down syndrome" [46].

\section{Theme 3: a life worth living}

The third theme was that of parents wanting to ensure their child's life would be a life worth living. Parents' decisions were influenced by their perception of what quality of life their child may encounter, both physically and emotionally, throughout their lives, as well as the impact caring for a child with difficulties would have on their own, and the quality of life of other members of their families (sub-theme 1). Some parents described their awareness that another child might not have these difficulties, and discussed their options of future healthy children as being influential in their decision-making process (sub-theme 2). Finally, a parent's own illness and competing responsibilities influenced their decision around ability to continue with a pregnancy affected by lethal, life-limiting, or severely debilitating conditions, with parents considering the impact this would have on already stretched resources (sub-theme 3 ).

Sub-theme 1: quality of life Parents considered the quality of life they anticipated their unborn child might have if they continued with a pregnancy, describing how this influenced their decision-making. Often parents reported that "they hoped that their child would remain comfortable throughout their life, without experiencing any pain or suffering" [54], considering the "likely extent and manageability of any physical or emotional suffering" [72]. Parents hoped that the child would be able to "feel or know that they were loved" [54], with some parents describing how they felt they could continue if "their baby could have a reasonable quality of life despite her/his condition" [72].

Other parents considered future implications on quality of life, and felt that they might find it "difficult to get treatment for this child" [77], with fears around how lack of treatment might impact on quality of life. Parents reflected on their child's future life, and how it may not be what they would hope for themselves, if they had been given a choice.

Often parents considered the impact continuing or terminating a pregnancy would have on their own quality of life. For some parents, they imagined that continuing with a pregnancy would increase the value of their lives, believing that raising a child with a lethal, lifelimiting, or severely debilitating condition would have brought them "closer together" as a family unit [54]. For other parents, the thought of the "emotional trauma" [65] of delivering and caring for a child who would eventually die was too much. Parents considered the risk to their "own health" [68] and "financial stability" [65], and how this might negatively impact their quality of life.

Parents also discussed the wider impact on wider family members' quality of life, with particular reference to other siblings who could be affected by the child's difficulties. Parents described how continuing with a pregnancy influenced their availability to support and guide their other children, considering the "added stress and burden" they could experience as a result of their unborn child's specific needs and difficulties [77]. Parents also considered potential future ramification for siblings burdened "with this child's care after they died" [65]. Some parents did describe how continuing with the pregnancy of a child 
with lethal, life-limiting, or severely debilitating disorders might add qualities to their other children's life, and looked forward with excitement for their children "to experience this" [77]. They described how a child with specific difficulties would "bring to light a little less selfishness and a little more giving" [77] to their family unit, improving their overall quality of life.

"We came to the conclusion that the baby had a heartbeat, and the reason she had a heartbeat was because I was her lifeline. This was a baby on life support, basically, and I was her life support, and that was a turning point in our decision. If we're in a horrible accident and a machine is pumping our blood and making us breathe and doing everything for us, we don't want to live like that. If it's good enough for us, why isn't it good enough for this baby" [58]?

Sub-theme 2: wait for alternate child Some parents anticipated future pregnancies and second chances at achieving the pregnancy they had hoped for, with an outcome they viewed as more favourable. Parents described wanting to "try again" [60] at creating a life which they had hoped for, and in turn achieve their "goals of having healthy children" [77].

For other parents, the association with birth and pregnancy as a joyful moment led them to consider whether the birth of a child with a lethal, life-limiting, or severely debilitating condition could change that. Some parents considered the impact this might have on their previous perception and "did not want to associate the beauty of delivery with suffering" [65].

Sub-theme 3: own illness and other responsibilities Finally, parents also described how their own illnesses had led them to consider the impact of a child with lifelimiting or lethal diagnosis, "because of the pressures of her own health condition" [72]. Similarly, some parents experienced their advanced age as a potential barrier to continuing with the pregnancy, describing how they were "thinking ahead" for the child's needs in later life [77]. Parents also described how other life responsibilities and the perceived pressure of a child with lethal, life-limiting, or severely debilitating conditions on their family unit impacted their decision-making.

"Other events such as illness in the family or unsettled home life impacted some participants ... and [at] that time our life was a bit of a mess because we'd sold the house and we were house sitting ... it was pretty horrendous for that whole time ... and still going on with the 10-month-old baby that we had and me being pregnant" [51].

\section{Discussion}

This review, the first to focus solely on parental decisionmaking following prenatal diagnosis of a lethal, lifelimiting, or severely debilitating conditions, augments our understanding of how parents arrive at a decision about their pregnancy. The review presents findings which support previous research suggesting that parents are influenced by values [17-21, 25], hope [25] and their own belief in their ability to care for a child with a lethal, lifelimiting, or severely debilitating diagnosis [25]. A previous review highlighted the importance of prior experience or connection to others with experience of disability [25], an anticipation of the quality of life for themselves and their unborn child [25], and the perception of their child as precious $[14,25]$. However, the current review also presents novel findings including those of the parents' imagined futures influencing their decision-making. Unlike previous reviews which reflect on how parents prepare themselves for potential setbacks in future pregnancies, this review describes how parents also reflecting on their ability to conceive another child in the future and their own personal factors which may influence this, such as advancing age. Overall, the process of decision-making for parents following prenatal diagnosis was complex and personalised, with parents reporting different areas of influence on their own decisions. Parents described longed for pregnancies which influenced their decision-making, whilst other parents described an attachment to their fetus as being critical when forming a choice regarding pregnancy options. The majority of parents also placed a high value on their own view of the child as a precious lifeform, which coincided with moral and fatalistic beliefs and values.

Whilst none of the previous reviews considered decision-making models, one paper within this review drew on problem-solving decisional models when reflecting on parental decision-making following diagnosis of lethal, life-limiting, or severely debilitating disorders $[79,80]$ The role of decision-making theory is an important aspect to consider when attempting to understand parental experiences. The sense that all life is precious appeared to be the key influencer of parents faced with a decision to terminate or continue pregnancies. The individual and nonquantifiable construct of an individual's belief in how worthwhile life is suggests that parents may well engage in more descriptive models of decision-making $[29,30]$, influenced by previous experiences and beliefs, rather than a more classical method of decision-making, whereby all potential outcomes are considered. Previous quantitative research had suggested that factors found within the main theme of 'All life is precious' are influential in parental decision-making, with the perceived fetus as precious [11] and attempts to do the right thing when making decisions [18-20]. The results from the current synthesis suggests 
that parents also consider personal life commitments, past experiences and fatalistic attitudes when considering whether to continue or terminate a pregnancy.

Whilst parental views of the importance of their fetus' life appeared to be the main influencer of parents' decisions, the current review also suggested that parents considered hope and the potential of the child's life being worth living when making their decision. This coincides with findings from a previous review evaluating decision-making within affected pregnancies [25] which also described how hope for a successful outcome, alongside consideration of expected quality of life was an important consideration for parents.

It should be acknowledged that this review identified only studies from countries in which termination was a legal and viable option for parents. Furthermore, the removal of two low quality papers means that not all existing literature was included.

\section{Clinical and research implications}

The current findings have important clinical implications for maternal healthcare teams supporting parents following diagnosis of lethal, life-limiting, or severely debilitating prenatal diagnosis as well as policy makers attempting to improve the overall care offered to parents during this time. Although this review highlights areas of shared importance for parents making these difficult decisions, it also emphasises the idiosyncratic nature of decision-making representative of descriptive models of decision-making $[29,30]$. Professionals supporting parents should enable discussion around individualised thoughts and beliefs during the decision-making process and ensure that they do not anticipate rigid or prescriptive responses based on the choices of other parents with similar demographics. As advanced age was viewed as a precursor to continuation of pregnancy for some parents and a factor in termination for others, providing an open and supportive environment in which to enable parents to explore their options and the factors important to them would be beneficial.

Care teams should, however, be aware of parents' beliefs around life, considering when they believe life begins, their attachment to the fetus, religious or moral values and any prior experiences which might have impacted their expectations of pregnancy or termination in order to support and scaffold parental decision-making and choice in a personalised and non-prescriptive manner.

Parents often struggled to accept the prognosis provided by medical professionals, doubting medical advice which did not facilitate hope, and wanting to conduct their own research to ensure there were no alternative pathways available to them. They also reported finding the experience of others helpful in alerting them to both hope of a life worth living, and the realistic difficulties they would face if continuing with their pregnancy.

Other parents considered the quality of life for their child and family unit when making their choice. Support could be provided by care teams facilitating contact with relevant groups and other parents who could provide insight into their own lived experience as a way of parents exploring this important factor themselves.

A tiered model of psychological care could be facilitated for parents following diagnosis of lethal, life-limiting, or severely debilitating disorders. The provision of tiered psychological care has been widely utilised within physical health care settings [81-83], with a multi-disciplinary team approach to providing psychological support to individuals experiencing emotional suffering. This could be provided through ward staff, and for a smaller number of parents struggling with adjustment or decision-making, through the provision of psychological practitioners such as Clinical Psychologists, in line with current recommendations for psychological care [84].

\section{Conclusion}

Whilst it has long been accepted that a parent's decisionmaking between termination or continuation of a pregnancy identified with a lethal, life-limiting, or severely debilitating condition is an emotionally traumatic and challenging experience [10], the specific influencers of decision-making were yet to be explored thoroughly. Given the consistency of themes found across all 22 included studies despite geographical differences, the findings appear to be applicable across different maternal healthcare systems in various countries. The current review highlights the importance of parents' perception of the fetus' life, basing their decision on moral beliefs and past-experience. It also suggests that some parents considered hope and the impact on their own and the fetus' life when making their decisions. Increasing the understanding of factors influencing parental decision-making will enable maternal healthcare professionals to provide an avenue for the exploration of these factors within a supportive environment and improve current services offered to parents following diagnosis.

\footnotetext{
Acknowledgements

We would like to express our gratitude to Gayathri Sundaresan, intern for Dr. Anja Wittkowski, for assisting with study inclusion selection and quality check.

\section{Authors' contributions}

CB assisted in the design of the study, conducted searches, screened titles, abstracts and full papers to identify eligible papers, conducted quality appraisal, extracted data, conducted meta-synthesis, constructed draft papers, contributed to the final draft and approved final draft. DMS assisted in the design of the study, extracted data, conducted meta-synthesis, constructed draft papers, contributed to the final draft and approved final paper. EJ assisted in the design of the study, constructed draft papers, contributed to the final draft and approved final paper. AW assisted in the initial development of the topic area and the design of the review, constructed draft papers,
} 
contributed to the final draft and approved final paper. AW oversaw the overall review process and any revisions.

\section{Funding}

No external funding was provided.

\section{Availability of data and materials}

All data generated or analysed during this study are included in this published article.

\section{Ethics approval and consent to participate}

Not applicable

\section{Consent for publication}

Not applicable

\section{Competing interests}

The authors declare that they have no competing interests.

\section{Author details}

${ }^{1}$ The University of Manchester, Faculty of Biology, Medicine and Health, School of Health Sciences, Division of Psychology and Mental Health, 2nd Floor, Zochonis Building, Brunswick Street, Manchester M13 9PL, UK. ²Greater Manchester Mental Health Foundation Trust, Trust Headquarters, Bury New Road, Prestwich, Manchester M25 3BL, UK. ${ }^{3}$ Leeds Trinity University, Brownberrie Lane, Horsforth LS18 5HD, Leeds, UK. ${ }^{4}$ Central Manchester University NHS Foundation Trust, Oxford Road, Manchester M13 9WL, UK.

\section{Received: 8 January 2019 Accepted: 29 July 2019}

\section{Published online: 08 August 2019}

\section{References}

1. World Health Organisation. Screening the Genes. World Health Organisation Bulletin. 2012;90(8):557-632

2. VanPutte AW. Perinatal bereavement crisis: coping with unexpected outcomes from prenatal diagnosis. J Perinat Neonatal Nurs. 1988;2(2):12-22.

3. Askelsdottir B, Conroy S, Rempel G. From diagnosis to birth: parents' experience when expecting a child with congenital anomaly. Adv Neonatal Care. 2008;8:348-54

4. Graham RH, Mason K, Rankin J, Robson SC. The role of feticide in the context of late termination of pregnancy: a qualitative study of health professionals and parent's views. Prenat Diagn. 2009;29:875-81. https://doi. org/10.1002/pd.2297.

5. Rauch ER, Smulian JC, DePrince K, Ananth CV, Marcella SW. New Jersey fetal abnormalities. Pregnancy interruption after second trimester diagnosis of fetal structural anomalies: the New Jersey fetal abnormalities registry. Am J Obstet Gynecol. 2005;193(4):1492-7. https://doi.org/10.1016/j.ajog.2005.02.099.

6. Drugan A, Johnson MP, Evans MI. Amniocentesis. In: Eden RD, Boehm FH editors. Assessment and Care of the Fetus: physiological, clinical, and medicolegal principles. Norwalk: Appleton and Lange; 1990. p. 283-90.

7. Hawkins A, Stenzel A, Taylor J, Chock V, Hudgins L. Variables influencing pregnancy termination following prenatal diagnosis of fetal chromosome abnormalities. J Genet Couns. 2012;22(2):238-48. https://doi.org/10.1007/s 0897-012-9539-1.

8. Shaffer BL, Caughey AB, Norton ME. Variation in the decision to terminate pregnancy in the setting of fetal aneuploidy. Prenat Diagn. 2006;26:667-71. https://doi.org/10.1002/pd.1462

9. Carlsson T, Starke V, Mattsson E. The emotional process from diagnosis to birth following a prenatal diagnosis of fetal abnormality: a qualitative study of messages in online discussion boards. Midwifery. 2017;1(48):53-9.

10. Quine L, Pahl J. First diagnosis of severe mental handicap: characteristics of unsatisfactory encounters between doctors and parents. Soc Sci Med. 1986; 22:53-62. https://doi.org/10.1016/0277-9536(86)90308-4.

11. Statham H, Solomou W, Chitty L. Prenatal diagnosis of fetal abnormality: psychological effects on women in low-risk pregnancies. Best Pract Res Clin Obstet Gynaecol. 2000;1(14):731-47.

12. Lotto R, Armstrong N, Smith LK. Care provision during termination of pregnancy following diagnosis of a severe congenital anomaly - a qualitative study of what is important to parents. Midwifery. 2016;43:14-20.

13. Black RB. Seeing the baby: the impact of ultrasound technology. J Genet Couns. 1992;1(4):5-54. https://doi.org/10.1007/BF00960084.
14. Draper J. "It was a real good show": the ultrasound scan, fathers and the power of visual knowledge. Sociol Health IIIn. 2002;24(6):771-95.

15. Hedrick JJ. The lived experience of pregnancy while carrying a child with a known, nonlethal congenital abnormality. Obstet Gynecol Neonatal Nurs. 2005;34(6):732-40. https://doi.org/10.1177/0884217505282020.

16. Schechtman KB, Gray DL, Baty JD, Rothman SM. Decision-making for termination of pregnancies with fetal anomalies: analysis of 53,000 pregnancies. Obstet Gynaecol. 2002;99(4):216-22. https://doi.org/10.1016/ S0029-7844(01)01673-8.

17. Verp M, Bombard A, Simpson J, Elias S. Parental decision following prenatal diagnosis of fetal chromosome abnormality. Am J Med Genet. 1988;29:61322. https://doi.org/10.1002/ajmg.1320290320.

18. Ahmed S, Green JM, Hewison J. Attitudes towards prenatal diagnosis and termination of pregnancy for thalassaemia in pregnant Pakistani women in the north of England. Prenat Diagn. 2006;26(3):248-57. https://doi.org/10.1 002/pd.1391.

19. Ngim C, Lai N, Ibrahim H, Ratnasingam V. Attitudes towards prenatal diagnosis and abortion in a multi-ethnic country: a survey among parents of children with thalassaemia major in Malaysia. J Community Genet. 2013; 4:215-21. https://doi.org/10.1007/s12687-012-0133-x.

20. Meryash D, Abuelo D. Counselling needs and attitudes toward prenatal diagnosis and abortion in fragile X families. Clin Genet. 1988;33:349-55.

21. Senanayake $H$, de Silva D, Premaratne S, Kulatunge M. Psychological reactions and coping strategies of Sri Lankan women carrying fetuses with lethal congenital malformations. Ceylon Med J. 2006;51:14-7.

22. Awwad R, Veach PM, Bartels DM, LeRoy BS. Culture and acculturation influences on Palestinian perceptions of prenatal genetic counselling. J Genet Couns. 2008:17(1):101-16.

23. Royal College of Obstetricians and Gynaecologists (RCOG). Termination of pregnancy for fetal abnormality in England, Scotland and Wales. 2010.

24. Royal College of Obstetricians and Gynaecologists (RCOG). Perinatal management of pregnant women at the threshold of infant viability (The obstetric perspective). 2014.

25. Sandelowski M, Barroso J. The travesty of choosing after positive prenatal diagnosis. J Obstet Gynecol Neonatal Nurs. 2005;34(3):307-18. https://doi. org/10.1177/0884217505276291.

26. Lafarge C, Mitchell K, Fox P. Termination of pregnancy for fetal abnormality: a meta-ethnography of women's experiences. Reprod Health Matters. 2014; 22(44):191-201. https://doi.org/10.1016/S0968-8080(14)44799-2.

27. Lou S, Jensen LG, Peterson OB, Vogel I, Hvidman L, Moller A, Nielsen CP. Parental response to severe or lethal prenatal diagnosis: a systematic review of qualitative studies. Prenat Diagn. 2017;37(8):731-43. https://doi.org/10.1002/pd.5093.

28. Beach LR, Lipshitz $R$. Why classical decision theory is an inappropriate standard for evaluating and aiding most human decision-making. In: Decision Making in Aviation, vol. 85; 2017.

29. Tversky A, Kahneman D. Advances in prospect theory: cumulative representation of uncertainty. J Risk Uncertain. 1992;5(4):297-323. https:// doi.org/10.1007/BF00122574

30. Falzer PR. Cognitive schema and naturalistic decision-making in evidencebased practices. J Biomed Inform. 2004;37(2):86-98. https://doi.org/10.1016/j. jbi.2004.02.002.

31. Lerner JS, Li Y, Valdesolo P, Kassam KS. Emotion and decision-making. Annu Rev Psychol. 2015:66:799-823. https://doi.org/10.1146/annurev-psych-010213-115043.

32. Vitell SJ, Nwachukwu SL, Barnes JH. The effects of culture on ethical decision-making: an application of Hofstede's typology. J Bus Ethics. 1993; 12(10):753-60. https://doi.org/10.1007/BF00881307.

33. Loewenstein $\mathrm{G}$, Lerner JS. The role of affect in decision-making. Handb Affect Sci. 2003;619(642):3.

34. McNeil BJ, Pauker SG, Sox HC Jr, Tversky A. On the elicitation of preferences for alternative therapies. N Engl J Med. 1982;306(21):1259-62.

35. Maule AJ, Hockey GRJ, Bdzola L. Effects of time-pressure on decision-making under uncertainty: changes in affective state and information processing strategy. Acta Psychol. 2000;104(3):283-301. https://doi.org/10.1016/S0001-6918(00)00033-0.

36. Shamseer L, Moher D, Clarke M, Ghersi D, Liberati A, Petticrew M, Shekelle $P$, Stewart LA. PRISMA-P group. BMJ. 2015;2:350.

37. Critical Appraisal Skills Programme: CASP Systematic Review Checklist, 2017 [online] Available at: https://casp-uk.net/wp-content/uploads/2018/01/CASPQualitative-Checklist-2018.pdf. Accessed 6 Aug 2018.

38. Health Evidence. Quality assessment tool: Review articles. http://www. healthevidence.org/documents/our-appraisal-tools/QA_tool\&dictionary_18. Mar.2013.pdf. Accessed 6 Aug 2018. 
39. Thomas J, Harden A. Methods for the thematic synthesis of qualitative research in systematic reviews. BMC Med Res Methodol. 2008;8:45. https:// doi.org/10.1186/1471-2288-8-45.

40. Boyatzis RE. Transforming qualitative information: thematic analysis and code development. Thousand Oaks: Sage publications; 1998.

41. Balkan M, Kalkanli S, Akbas H, Yalinkaya A, Nail Alp M, Budak T. Parental decisions regarding a prenatally detected fetal chromosomal abnormality and the impact of genetic counselling: an analysis of 38 cases with aneuploidy in Southeast Turkey. J Genet Couns. 2010;19:241-6.

42. Fleming V, Iljuschin I, Pehlke-Milde J, Maurer F, Parpan F. Dying at life's beginning: experiences of parents and health professionals in Switzerland when an 'in utero' diagnosis incompatible with life is made. J Midwifery. 2016;34:23-9. https://doi.org/10.1016/j.midw.2016.01.014.

43. Huyard C. Decision-making after prenatal diagnosis of a syndrome predisposing to intellectual disability: what prospective parents need to know and the importance of non-medical information. J Intellect Develop Disabil. 2012;37(4):315-23. https://doi.org/10.3109/13668250.2012.726350.

44. Timstra TJ, Bosboom GJM, Bouman K. Ervaringen van vrouwen die besloten de zwangerschap uit te dragen na diagnose 'Down-syndroom'. NTG. 2000; 144(44):2104-7.

45. Lenhard W, Ebert $H$, Breitenbach E, Schindelhauer D, Deutscher H, Henn W. Basic conditions for the continuation of a pregnancy in expectance of a child with Down syndrome. Vierteljahresschrift fur Heilpadagogik und ihre Nachbargebiete. 2006;75(1):40-50

46. Helm DT, Miranda S, Angoff CN. Prenatal diagnosis of Down syndrome: mothers' reflections on supports needed from diagnosis to birth. Ment Retard. 1998;36(1):55-61.

47. Redlinger-Grosse K, Bernhardt BA, Berg K, Muenke M, Biesecker BB. The decision to continue: the experiences and needs of parents who receive a prenatal diagnosis of Holoprosencephaly. Am J Med Genet. 2002;112:36978. https://doi.org/10.1002/ajmg.10657.

48. Silverman D. Interpreting qualitative data: methods for analysing talk, text and interaction. London: Sage; 1993.

49. Rempel GR, Cender LM, Lynam MJ, Sandor GG, Farquharson D. Parents' perspectives on decision-making after antenatal diagnosis of congenital heart disease. J Obstet Gynecol Neonatal Nurs. 2004;33(1):64-70.

50. Blumer H. Symbolic interactionism: perspective and method. Englewood Cliffs: Prentice Hall; 1969.

51. Hickerton $\mathrm{CL}$, Aitken M, Hodgson J, Delatycki MB. "Did you find that out in time?": new life trajectories of parents who choose to continue a pregnancy where a genetic disorder is diagnosed or likely. Am J Med Genet. 2012;158: 373-83. https://doi.org/10.1002/ajmg.a.34399.

52. Glasser BG, Strauss AL. The discovery of grounded theory: strategies for qualitative research. New Brunswick: Aldine Transaction; 1967.

53. Hurford E, Hawkins A, Hudgins L, Taylor J. The decision to continue a pregnancy affected by Down syndrome: timing of decision and satisfaction with receiving a prenatal diagnosis. J Genet Couns. 2013;22(5):587-93.

54. Guon J, Wilfond BS, Farlow B, Brazg T, Janvier A. Our children are not a diagnosis: the experience of parents who continue their pregnancy after prenatal diagnosis of trisomy 13 or 18. Am J Med Genet. 2014;164(2):30818. https://doi.org/10.1002/ajmg.a.36298.

55. Gesser-Edelsburg A, Shahbari NAE. Decision-making on terminating pregnancy for Muslim Arab women pregnant with foetuses with congenital anomalies: maternal affect and doctor-patient communication. J Reprod Health. 2017;14:49-61.

56. Strauss A, Corbin J. Basics of qualitative research, techniques and procedures for developing grounded theory. 2nd ed. Thousand Oaks: Sage Publications; 1998.

57. Moudi Z, Miri-Moghaddam E. Decisions regarding pregnancy termination due to Beta-thalassemia major: a mixed methods study in Sistan and Baluchestan. Iran J Genet Couns. 2017;26:556.

58. Bryar SH. One day you're pregnant and one day you're not: pregnancy interruption for fetal anomalies. J Obstet Gynecol Neonatal Nurs. 1997;16(5):559-66.

59. Swanson-Kauffman K, Schonwald E. Phenomenology. NLN Publ. 1998; 15(2233):97-105

60. Ferreira da Costa LD, Hardy E, Osis MJD. Termination of pregnancy for fetal abnormality incompatible with life: Women's experiences in Brazil. Reprod Health Matters. 2005;13(26):139-46.

61. Minayo MCS. O desafio do conhecimento: pesquisa qualitativa em saú de. In: Rio de Janeiro7 Hucitec-Abrasco. 7th ed; 2000. p. 197-247.

62. Leichtentritt RD. Silenced voices: Israeli mothers' experience of feticide. J Soc Sci Med. 2011;72:747-54. https://doi.org/10.1016/j.socscimed.2010.12.021.
63. Gubrium JF, Holstein JA. At the border of narrative and ethnography. J Contemp Ethnogr. 1999;28(5):561. https://doi.org/10.1177/08912419912 9023550.

64. Benute GRG, Nomura RMY, Liao AW, de Lourdes BM, Lucia MCS, Zugaib M. Feelings of women regarding end-of-life decision-making after ultrasound diagnosis of a lethal malformation. J Midwifery. 2012;18:472-5. https://doi. org/10.1016/j.midw.2011.06.011.

65. Gawron LM, Cameron KA, Phisuthikul A, Simon MA. An exploration of women's reasons for termination timing in the setting of fetal abnormalities. J Contracept. 2013;88:109-15.

66. Ioannou L, Delatycki MB, Massie J, Hodgson J, Lewis S. "Suddenly having two positive people who are carriers is a whole new thing" - experiences of couples both identified as carriers of cystic fibrosis through a population-based carrier screening program in Australia. J Genet Couns. 2015;24:987-1000.

67. Elo S, Kyngas H. The qualitative content analysis process. J Adv Nurs. 2008; 62(1):107-15. https://doi.org/10.1111/j.1365-2648.2007.04569.x.

68. Sandelowski M, Jones LC. Healing fictions': stories of choosing in the aftermath of the detection of fetal abnormalities. Soc Sci Med. 1996;42(3):353-61.

69. Miles MB, Huberman AM. Qualitative data analysis: an expanded sourcebook. 2nd ed. Thousand Oaks: Sage Publications; 1994

70. Locock L, Alexander J. 'Just a bystander'? Men's place in the process of fetal screening and diagnosis. J Soc Sci Med. 2006:62:1349-59.

71. Pope C, Ziebland S, Mays N. Qualitative research in health care: Analysing qualitative data. Br Med J. 2000;320:114-6. https://doi.org/10.1136/bmj.320. 7227.114.

72. France EF, Locock L, Hunt K, Ziebland S, Field K, Wyke S. Imagined futures: how experiential knowledge of disability affects parents' decision-making about fetal abnormality. Health Expect. 2012;15(2):139-56. https://doi.org/1 0.1111/j.1369-7625.2011.00672.x.

73. Bury M. IIIness narratives: fact or fiction? Sociol Health IIIn. 2001;23(3):263-85. https://doi.org/10.1111/1467-9566.00252.

74. Lawson KL, Pierson RA. Maternal decisions regarding prenatal diagnosis: rational choices or sensible decisions? J Obstet Gynaecol Can. 2007;29:2406. https://doi.org/10.1016/S1701-2163(16)32412-4.

75. Hodgson J, Pitt P, Metcalfe S, Halliday J, Menezes M, Fisher J, Hickerton C, Peterson K, McClaren B. Experiences of prenatal diagnosis and decisionmaking about termination of pregnancy: a qualitative study. Aust N Z Obstet Gynaecol. 2016;56(6):605-13. https://doi.org/10.1111/ajo.12501.

76. Braun V, Clarke V. Using thematic analysis in psychology. Qual Res Psychol. 2005;3:77-101. https://doi.org/10.1191/1478088706qp063oa.

77. Reed AR, Berrier KL. A qualitative study of factors influencing decisionmaking after prenatal diagnosis of Down syndrome. J Genet Couns. 2017; 26:814-28. https://doi.org/10.1007/s10897-016-0061-8.

78. Levers MJ. Philosophical paradigms, grounded theory, and perspectives on emergence. Sage Open. 2013;16(3):4. https://doi.org/10.1177/215824401351 7243

79. Rettig KD. Problem-solving and decision-making as a central process of family life: an ecological framework for family relations and family resource management. In: Settles BH, Hanks RS, Sussman MB, editors. American families and the future: analysis of possible destinies. New York: Hawarth; 1993. p. 187.

80. Tallman I. Theoretical issues in reaching problem solving in families. In: Settles BH, Hanks RS, Sussman MB, editors. American families and the future: Analysis of possible destinies. New York: Hawarth; 1993. p. 155.

81. Kazak AE. Pediatric Psychosocial Preventative Health Model (PPPHM): research, practice, and collaboration in pediatric family systems medicine. Fam Syst Health. 2006;24(4):381.

82. Wisely JA, Hoyle E, Tarrier N, Edwards J. Where to start?: attempting to meet the psychological needs of burned patients. Burns. 2007;33(6):736-46.

83. Zeiss AM, Karlin BE. Integrating mental health and primary care services in the Department of Veterans Affairs health care system. J Clin Psychol Med Settings. 2008;15(1):73-8.

84. National institute for health and care excellence. Common mental health problems: identification and pathways to care. 2011.

\section{Publisher's Note}

Springer Nature remains neutral with regard to jurisdictional claims in published maps and institutional affiliations. 